ACCEPTED FOR PUBLiCATion IN APJS

Preprint typeset using $\mathrm{LAT}_{\mathrm{E}} \mathrm{X}$ style emulateapj v. 8/13/10

\title{
THE K2 ECLIPTIC PLANE INPUT CATALOG (EPIC) AND STELLAR CLASSIFICATIONS OF 138,600 TARGETS IN CAMPAIGNS 1-8
}

\author{
Daniel Huber ${ }^{1,2,3}$, Stephen T. Bryson ${ }^{4}$, Michael R. HaAs ${ }^{4}$, Thomas Barclay ${ }^{4,5}$, Geert Barentsen ${ }^{4,5}$ \\ Steve B. Howell ${ }^{4}$, Sanjib Sharma ${ }^{1}$, Dennis Stello ${ }^{1,3}$, and Susan E. Thompson ${ }^{2,4}$ \\ accepted for publication in ApJS
}

\begin{abstract}
The K2 Mission uses the Kepler spacecraft to obtain high-precision photometry over $\approx 80$ day campaigns in the ecliptic plane. The Ecliptic Plane Input Catalog (EPIC) provides coordinates, photometry and kinematics based on a federation of all-sky catalogs to support target selection and target management for the K2 mission. We describe the construction of the EPIC, as well as modifications and shortcomings of the catalog. Kepler magnitudes $(K p)$ are shown to be accurate to $\approx 0.1 \mathrm{mag}$ for the Kepler field, and the EPIC is typically complete to $K p \approx 17$ ( $K p \approx 19$ for campaigns covered by SDSS). We furthermore classify 138,600 targets in Campaigns $1-8(\approx 88 \%$ of the full target sample) using colors, proper motions, spectroscopy, parallaxes, and galactic population synthesis models, with typical uncertainties for G-type stars of $\approx 3 \%$ in $T_{\text {eff }}, \approx 0.3 \operatorname{dex}$ in $\log g, \approx 40 \%$ in radius, $\approx 10 \%$ in $\mathrm{mass}$, and $\approx 40 \%$ in distance. Our results show that stars targeted by $\mathrm{K} 2$ are dominated by $\mathrm{K}-\mathrm{M}$ dwarfs $(\approx 41 \%$ of all selected targets $), \mathrm{F}-\mathrm{G}$ dwarfs $(\approx 36 \%)$ and $\mathrm{K}$ giants $(\approx 21 \%)$, consistent with key K2 science programs to search for transiting exoplanets and galactic archeology studies using oscillating red giants. However, we find a significant variation of the fraction of cool dwarfs with galactic latitude, indicating a target selection bias due to interstellar reddening and the increased contamination by giant stars near the galactic plane. We discuss possible systematic errors in the derived stellar properties, and differences to published classifications for $\mathrm{K} 2$ exoplanet host stars. The EPIC is hosted at the Mikulski Archive for Space Telescopes (MAST): http://archive.stsci.edu/k2/epic/search.php Subject headings: catalogs — planetary systems — proper motions — stars: fundamental parameters — stars: late-type - techniques: photometric
\end{abstract}

\section{INTRODUCTION}

The NASA Kepler Mission (Borucki et al. 2010; Koch et al. 2010) delivered breakthrough discoveries in exoplanet science and stellar astrophysics by obtaining high precision photometry of a single field for four years. To facilitate target selection and to enable the definition of optimal apertures for the extraction of light curves, a dedicated ground-based imaging campaign of the Kepler field was federated with available all-sky catalogs to create the Kepler Input Catalog (KIC, Brown et al. 2011), which contains $\approx 13$ million sources centered on the Kepler field. In addition to broadband photometry the KIC includes Kepler magnitudes $(K p)$, which were defined using gri magnitudes through a calibration of the expected flux in the Kepler bandpass, and stellar properties derived from fitting photometry to synthetic colors from model atmospheres. The KIC has been an indispensable tool for a variety of Kepler science such as target selection (Batalha et al. 2010), planet-candidate catalogs (Borucki et al. 2011a,b;

\footnotetext{
${ }^{1}$ Sydney Institute for Astronomy (SIfA), School of Physics, University of Sydney, NSW 2006, Australia; daniel.huber@sydney.edu.au

2 SETI Institute, 189 Bernardo Avenue, Mountain View, CA 94043, USA

${ }^{3}$ Stellar Astrophysics Centre, Department of Physics and Astronomy, Aarhus University, Ny Munkegade 120, DK-8000 Aarhus C, Denmark

${ }^{4}$ NASA Ames Research Center, Moffett Field, CA 94035, USA

${ }^{5}$ Bay Area Environmental Research Inst., 560 Third St., West Sonoma, CA 95476, USA
}

Batalha et al. 2013), studies of planet occurrence rates (Youdin 2011; Howard et al. 2012; Fressin et al. 2013), and the derivation of fundamental properties of oscillating stars (Kallinger et al. 2010; Chaplin et al. 2011).

Following the failure of the second of four reaction wheels, the Kepler mission ended in May 2013 due to the inability to maintain fine-point in the original field. The spacecraft was subsequently repurposed as the K2 mission to observe fields along the ecliptic plane using the solar pressure to maintain fine-point along the roll axis of the spacecraft (Howell et al. 2014). Methodologies to account for systematic variability caused by the spacecraft thruster firings are continuously being developed (Vanderburg \& Johnson 2014; Aigrain et al. 2015; Angus et al. 2016; Huang et al. 2015; Lund et al. 2015) and have demonstrated that the photometric precision of $\mathrm{K} 2$ is at least within a factor of 2 of Kepler. Early K2 science results include the discovery of exoplanets around bright GKM dwarfs (Crossfield et al. 2015; Vanderburg et al. 2015; Foreman-Mackey et al. 2015; Petigura et al. 2015), eclipsing binary stars (Armstrong et al. 2015; LaCourse et al. 2015), as well as the detection of pulsations across the HR diagram such as subgiants (Chaplin et al. 2015), red giants (Stello et al. 2015), white dwarfs (Hermes et al. 2014), RR Lyrae stars (Molnár et al.|2015; Kurtz et al. 2016), O stars (Buvsschaert et al. 2015) and subdwarf B stars (Jeffery \& Ramsay 2014).

In this paper we describe the Ecliptic Plane Input Catalog (EPIC) for the K2 mission, which serves a similar role as the KIC for the Kepler mission. The primary 
purpose of the catalog is to provide positions and Kepler magnitudes for target management and the definition of optimal apertures. A secondary goal is to provide observables to facilitate target selection by the community. The EPIC does not contain stellar properties for all sources, but in this work we perform classifications of 138,600 targets which were selected for observations to support community investigations of K2 data. Since K2 field positions are not fixed for the duration of the mission, the EPIC is continuously updated to include new campaign fields. At the time of writing of this paper the EPIC has been released for Campaigns 1-13 (C1-C13), with no substantial changes expected for future fields.

\section{CATALOG CONSTRUCTION}

\subsection{Input Sources and Cross-Matching}

EPIC sources are drawn from several publicly available all-sky catalogs: the Hipparcos catalog van Leeuwen 2007), the Tycho-2 catalog (Høg et al. 2000), the fourth US Naval Observatory CCD Astrograph Catalog (UCAC4, Zacharias et al. 2013), the Two Micron All Sky Survey (2MASS, Skrutskie et al. 2006), and data release 9 of the Sloan Digital Sky Survey (SDSS DR9, Ahn et al. 2012). Each catalog was downloaded from Vizier a search radius of 9 degrees centered on each $\mathrm{K} 2$ campaign field, which is large enough to allow for potential small adjustments to the field of view after the EPIC is made available to the community.

The quality of the photometry in the input catalogs varies significantly. To ensure some homogeneity, the following quality cuts and transformations were applied to the observables:

- Tycho-2: $B_{T}$ and $V_{T}$ photometry were converted into the Johnson $B V$ by interpolating Table 2 in Bessell (2000).

- UCAC4: $g^{\prime} r^{\prime} i^{\prime}$ photometry (which originates from the AAVSO Photometric All-Sky Survey, APASS7) was converted to the Sloan gri system using the transformation equations given on the SDSS website. APASS $B V$ gri uncertainties which are zero or $0.01 \mathrm{mag}$ in UCAC4 were replaced with average APASS uncertainties as a function of $B V g r i$ magnitude.

- 2MASS: All sources brighter than $J=5$ mag were discarded due to known saturation problems, and all sources with a $J$-band quality flag worse than $\mathrm{C}$ were removed. All $H$ and $K$-band measurements were also omitted for quality flags worse than C.

- SDSS DR9: Only targets with clean photometry, $r$ magnitudes lower than 20, and photometry errors lower than 0.5 mag were retained.

Each catalog was cross-matched for overlapping sources. Published matches (Hipparcos-Tycho, UCAC-Tycho2MASS) were adopted when available, otherwise sources were matched by finding the closest object within 3 arcseconds. To eliminate chance matches with background

6 http://webviz.u-strasbg.fr/viz-bin/VizieR

7 http://www.aavso.org/apass

8 http://www.sdss.org/dr7/algorithms/jpeg_photometric_eq_dr1.html http://archive.stsci.edu/cgi-bin/dss_form
TABLE 1

Ecliptic Plane Input Catalog columns.

\begin{tabular}{lll}
\hline Name & Unit & Description \\
\hline EPIC & none & K2 Identifier \\
HIP & none & Hipparcos Identifier \\
TYC & none & Tycho-2 Identifier \\
UCAC & none & UCAC4 Identifier \\
2MASS & none & 2MASS Identifier \\
SDSS & none & SDSS Identifier \\
Object type & none & Object Type Flag \\
& & [STAR, EXTENDED] \\
Kepflag & none & Kepler Magnitude Flag \\
& & [gri, BV, JHK, J] \\
RA & degrees & Right Ascension (JD2000) \\
Dec & degrees & Declination (JD2000) \\
pmra & mas/yr & Proper Motion in RA \\
pmdec & mas/yr & Proper Motion in DEC \\
plx & mas & Parallax \\
Bmag & magnitude & Johnson B band magnitude \\
Vmag & magnitude & Johnson V band magnitude \\
umag & magnitude & Sloan u band magnitude \\
gmag & magnitude & Sloan g band magnitude \\
rmag & magnitude & Sloan r band magnitude \\
imag & magnitude & Sloan i band magnitude \\
zmag & magnitude & Sloan z band magnitude \\
Jmag & magnitude & 2MASS J band magnitude \\
Hmag & magnitude & 2MASS H band magnitude \\
Kmag & magnitude & 2MASS K band magnitude \\
KepMag & magnitude & Kepler magnitude (Kp) \\
\hline NOMAD & none & NOMAD1 Identifier \\
Mflg & none & 2MASS Flags \\
& & [Qflg-Rflg-Bflg-Cflg-Xflg-Aflg] \\
proxy & as & 2MASS nearest neighbor \\
\hline & & \\
Mmag & &
\end{tabular}

Notes: Bracketed items list the possible values for catalog flags (see text for details). Columns containing uncertainties and columns which are typically not populated for any given EPIC source (such as stellar properties) are omitted from this table for clarity.

objects, the $V$ or $g$ magnitude of the target and matched source were required to agree within $1.5 \mathrm{mag}$, which is the typical maximum $3 \sigma$ uncertainty in a given passband. For cross-matches without common passbands (such as Tycho-2MASS, or 2MASS-SDSS), the $V$ and $g$ magnitudes of the matched source were estimated using Johnson-2MASS-SDSS transformations of Bilir et al. (2005) and Bilir et al. (2008). For transformed magnitudes, the matching criteria were conservatively set to 2 mag for $g$-band magnitudes estimated from $B V$, and 4 mag for $g$-band magnitudes estimated from $J H K$. While this procedure should eliminate most erroneous crossmatches, it may result in duplicate entries. Visual inspection of images from the second Palomar Observatory Sky Survey (POSS-II) 9 showed that duplicate entries are relatively rare (see also Figure 4).

For each source, the following observables were cataloged (see Table 1): Right Ascension (RA), Declination (DEC), proper motion in RA, proper motion in DEC, parallax, Johnson $B V, 2 \mathrm{MASS} J H K$, Sloan ugriz, and 

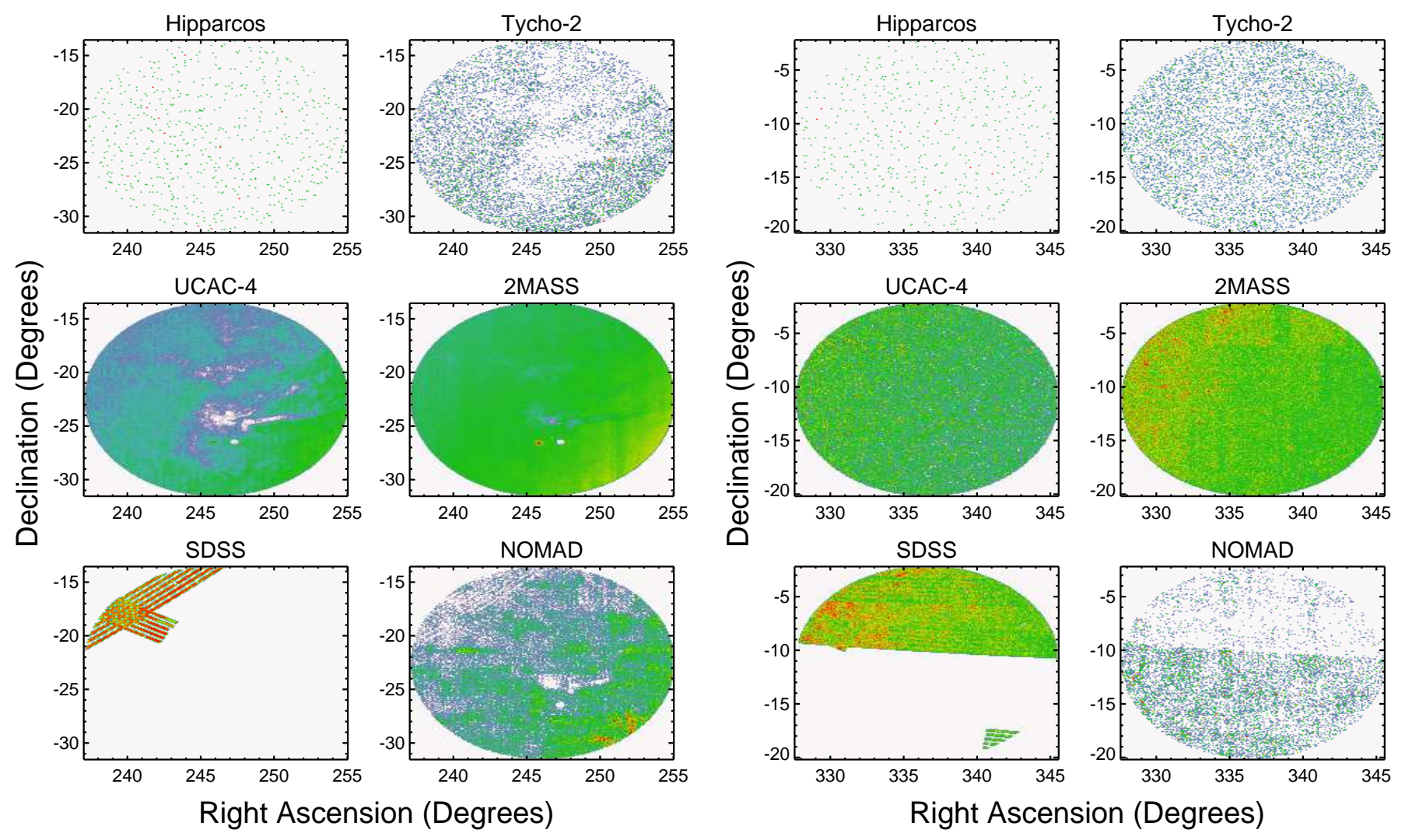

FIG. 1.- Distribution of EPIC sources in C2 (left) and C3 (right) subdivided into the different input catalogs. Color coding denotes logarithmic number density in each field. Note that the lack of sources near the field center for C2 is due to dust extinction from the $\rho$ Oph star forming region. The hole at $\mathrm{RA} \approx 247.4$ degrees and $\mathrm{Dec} \approx-26.4$ degrees is caused by $\alpha$ Sco $(V \approx 0.9$ mag). The bimodal distribution in the bottom right panel for $\mathrm{C} 3$ is caused by the fact that NOMAD proper motions are only used for 2MASS cross-matched sources that do not have proper motions listed in Tycho, UCAC or SDSS.

associated uncertainties. For sources with photometry in multiple catalogs, Tycho $B V$ and UCAC gri were prioritized over APASS $B V$ and SDSS gri to avoid saturation problems for bright stars. Cross-matched identifiers of all catalogs were also recorded. Additionally, extended sources identified in either 2MASS or SDSS were flagged in the "Objtype" identifier.

Following community advocacy, starting with $\mathrm{C} 2$ the EPIC was supplemented with proper motions from the NOMAD1 catalog (Zacharias et al. 2005) to increase completeness for faint high-proper motion stars. NOMAD values were only used for 2MASS cross-matched sources for which no other proper motions were available (i.e., NOMAD does not override Tycho, UCAC, or SDSS). Additionally, 2MASS quality and proximity flags were added. We refer the reader to Skrutskie et al. (2006) for the documentation on these flags.

Figure 1 shows the sky coverage of each input catalog for C2 and C3. Note that SDSS DR9 frequently does not cover the entire field, and hence the completeness of the EPIC may vary within a given campaign. Additionally, individual catalogs may show local incompleteness due to bright stars or regions with high extinction, which are clearly visible in Figure 1 for the optical catalogs in C2.

\subsection{EPIC ID Assignment}

Each catalog source is assigned a unique EPIC number, which serves as the K2 identifier. Table 2 lists EPIC IDs assigned for sources from $\mathrm{C} 0-\mathrm{C} 13$. Catalogs are divided into 2 types: "Comprehensive catalogs" are base catalogs for each campaign, and EPIC IDs are assigned with increasing declination. "Missing targets" are sources that were proposed for $\mathrm{K} 2$ observations by the community but were not present in the comprehensive catalog, for example due to being fainter than the completeness limit. For such cases, the K2 Science Office assigned new EPIC IDs and added the sources to the catalog for target management. We note that such sources only have coordinates and Kepler magnitudes as provided by the community, but lack ancillary information such as magnitudes and cross-matched identifiers that are typically available for other EPIC sources.

The EPIC ID bears no information on the campaign in which a target has been observed. This is due to the fact that K2 will re-observe parts of some fields, and hence a given target can be contained in more than one campaign. EPIC IDs start at 201 Million, while K2 custom aperture targets have IDs between 200 million and 201 million. Table 3 provides an overview of the ID ranges used for different targets in the Kepler and K2 missions.

\section{KEPLER MAGNITUDES}

Kepler magnitudes $(K p)$ are estimated using observed broadband magnitudes. To place K2 $K p$ values as closely as possible to Kepler $K p$ values, we follow the definitions using gri photometry in Equations (2)-(5) by Brown et al. (2011). However, since gri photometry is not available for every source, different methods had to 
TABLE 2

LOG OF EPIC IDS ASSIGNED BY CAMPAIGN

\begin{tabular}{|c|c|c|c|c|c|}
\hline Delivery Date & Campaign & Catalog Type & First EPIC ID & Last EPIC ID & Number of Targets \\
\hline $02 / 05 / 14$ & $\mathrm{C} 1$ & Comprehensive & 201000001 & 202059065 & 1059065 \\
\hline $02 / 10 / 14$ & $\mathrm{CO}$ & Proposed Targets & 202059066 & 202154323 & 95258 \\
\hline $04 / 07 / 14$ & $\mathrm{C} 2$ & Comprehensive & 202154324 & 205871527 & 3717204 \\
\hline $04 / 07 / 14$ & C3 & Comprehensive & 205871528 & 206598205 & 726678 \\
\hline $04 / 18 / 14$ & $\mathrm{CO}$ & Comprehensive & 206598206 & 210282463 & 3684258 \\
\hline $04 / 25 / 14$ & $\mathrm{C} 1$ & Missing Targets & 210282464 & 210282491 & 28 \\
\hline $07 / 03 / 14$ & $\mathrm{C} 2$ & Missing Targets & 210282492 & 210282560 & 69 \\
\hline $07 / 03 / 14$ & $\mathrm{C} 4$ & Comprehensive & 210282561 & 211233315 & 950755 \\
\hline $07 / 03 / 14$ & C5 & Comprehensive & 211233316 & 212235315 & 1002000 \\
\hline 09/09/14 & C3 & Missing Targets & 212235316 & 212235356 & 41 \\
\hline 09/14/14 & C6 & Comprehensive & 212235357 & 212886326 & 650970 \\
\hline $09 / 17 / 14$ & $\mathrm{C} 7$ & Comprehensive & 212886327 & 220115896 & 7229570 \\
\hline $03 / 30 / 15$ & $\mathrm{C} 4$ & Missing Targets & 220115897 & 220115973 & 77 \\
\hline $03 / 30 / 15$ & $\mathrm{C} 8$ & Comprehensive & 220115974 & 220769262 & 653289 \\
\hline $03 / 30 / 15$ & C9 & Comprehensive & 220769263 & 228682308 & 7913046 \\
\hline $03 / 30 / 15$ & C5 & Missing Targets & 228682309 & 228683400 & 1092 \\
\hline $03 / 30 / 15$ & $\mathrm{C} 10$ & Comprehensive & 228683401 & 229227137 & 543737 \\
\hline $12 / 03 / 15$ & $\mathrm{C} 6$ & Missing Targets & 229227138 & 229228144 & 1007 \\
\hline $12 / 03 / 15$ & $\mathrm{C} 7$ & Missing Targets & 229228145 & 229228373 & 229 \\
\hline $12 / 03 / 15$ & $\mathrm{C} 8$ & Missing Targets & 229228374 & 229228998 & 625 \\
\hline $12 / 03 / 15$ & C11 & Comprehensive & 229228999 & 245899083 & 16670085 \\
\hline $12 / 03 / 15$ & $\mathrm{C} 12$ & Comprehensive & 245899084 & 246541209 & 642126 \\
\hline $12 / 03 / 15$ & $\mathrm{C} 13$ & Comprehensive & 246541210 & 248368655 & 1827446 \\
\hline
\end{tabular}

Notes: "Comprehensive" are base catalogs including all information listed in Table 1 "Proposed Targets" and "Missing Targets" are sources generated based on community-provided information and hence do not include catalog cross-matched information such as alternate identifiers and photometry (see Section 4.1 for details). Note that the completeness of the EPIC in C9 is reduced due to the high source density (see Section 4.6).

TABLE 3

IDENTIFIERS USED By the KePler ANd K2 Missions

\begin{tabular}{ll}
\hline ID & Targets \\
\hline $0-30 \mathrm{M}$ & KIC Kepler Catalog Targets) \\
30M-60M & UKIRT KIC extension targets \\
$60 \mathrm{M}-100 \mathrm{M}$ & K2 engineering test targets \\
100M-200M & Kepler Custom Aperture Targets \\
$200 \mathrm{M}-201 \mathrm{M}$ & K2 Custom Aperture Targets \\
$>201 \mathrm{M}$ & EPIC (K2 Catalog Targets) \\
\hline
\end{tabular}

be adopted. The EPIC identifier "KepFlag" keeps track of which photometry was used to calculate the Kepler magnitude. The following prioritization was adopted (brackets denote the Kepflag string listed in Table 1):

- Kepflag = [gri]: Kp was calculated from gri magnitudes using Equations (2)-(5) in Brown et al. (2011).

- Kepflag $=[\mathrm{BV}]$ : Sloan $g r$ was estimated from Johnson $B V$ using the transformations by Bilir et al. (2005):

$$
g-r=1.124(B-V)-0.252
$$

and

$$
g=V+0.634(B-V)-0.108 .
$$

$K p$ was then calculated from $g r$ using Equation (2) in Brown et al. (2011).
- Kepflag $=[\mathrm{JHK}]: K p$ was calculated using the polynomial $J-K$ relations by Howell et al. (2012). Given $x=J-K$ these transformations are:

$$
\begin{array}{r}
K p=0.42443603+3.7937617 x-2.3267277 x^{2} \\
+1.4602553 x^{3}+K
\end{array}
$$

for all stars with $J-H>0.75$ and $H-K>0.1$ (approximate color cut for giants), and

$$
\begin{array}{r}
K p=0.314377+3.85667 x+3.176111 x^{2}- \\
25.3126 x^{3}+40.7221 x^{4} \\
-19.2112 x^{5}+K,
\end{array}
$$

for all remaining stars. The above relations are applied for $-0.2<J-K<1.2$ for giants and $-0.2<J-K<$ 1.0 for dwarfs, which are the calibration ranges given by Howell et al. (2012).

- Kepflag $=[\mathrm{J}]$ : For sources outside the color limits of Equations (3) and (4) or sources which only have a valid $J$-band magnitude, a rough estimate of $K p$ was calculated from the $J$-band magnitude using the rela- 
tions by Howell et al. (2012):

$$
\begin{array}{r}
K p=-398.04666+149.08127 J-21.952130 J^{2}+ \\
1.5968619 J^{3}-0.057478947 J^{4} \\
+0.00082033223 J^{5}+J
\end{array}
$$

for $J=10-16.7$ and

$$
K p=0.1918+1.08156 J
$$

for $J>16.7$.

For bright near-infrared sources $(J<10)$ which do not have optical photometry and fall outside the $J(H K)$ calibration range in Howell et al. (2012), Kepler magnitudes were assumed to be equal to their $J$-band magnitude for $\mathrm{C} 1-\mathrm{C} 7$. Starting with $\mathrm{C} 8$, this criterium was changed to $K p=J+1.7$ to avoid a discontinuity in Kepler magnitudes for highly reddened fields in which a significant fraction of sources lack optical photometry and fall outside the calibration range (see Figure 18 in Howell et al. 2012). We note that this discontinuity only affects $<0.1 \%$ of all sources in the EPIC for $\mathrm{C} 1-\mathrm{C} 7$.

Figure 2 compares Kepler magnitudes calculated from UCAC gri, APASS $B V, 2 \mathrm{MASS} J H K$, and 2MASS $J$ to original $K p$ values for a random sample of 5000 targets in the original Kepler field. The values show good agreement for the first three methods (panels a-c), with a median offset and scatter of $-0.01 \pm 0.09$ mag for UCAC gri, $-0.03 \pm 0.13 \mathrm{mag}$ for APASS $B V$, and $0.01 \pm 0.12 \mathrm{mag}$ for 2MASS $J H K$. Users should be aware that Kepler magnitudes based on $J$ (panel d) are very approximate since the transformation is based on the average colors of stars in the Kepler field. Deviations of up to $1 \mathrm{mag}$ can be observed for very blue or red sources.

Figure 3 shows the Kepler magnitude distribution for sources in $\mathrm{a} \approx 80$ square degree field in $\mathrm{C} 1$ covered by 2 MASS and SDSS. The steep drop-off at $\mathrm{Kp} \approx 20 \mathrm{mag}$ is caused by the $r<20 \mathrm{mag}$ cut in SDSS DR9. For regions not covered by SDSS the completeness is set by 2MASS. Panel (b) of Figure 3 shows the distribution on a logarithmic scale, illustrating that the catalog includes a small number of objects with $K p>25$, which are predominantly galaxies identified in SDSS.

Figure 3 and visual inspection of POSS-II images showed that the EPIC is complete to about $K p \approx 19$ for areas covered by SDSS, and $K p \approx 17$ for areas covered by 2MASS only. Completeness for fields only covered by 2MASS may be significantly reduced for sources that are blue and faint.

\section{CATALOG ADJUSTMENTS AND KNOWN SHORTCOMINGS}

\subsection{Merging and Overrides for Missing Targets}

Targets which were proposed for observations by the community but not matched with an existing EPIC ID are reconciled with the comprehensive catalog for each campaign to avoid source duplication and consequent complications for computing source crowding, flux fraction, and optimal apertures (see Thompson \& Fraquelli
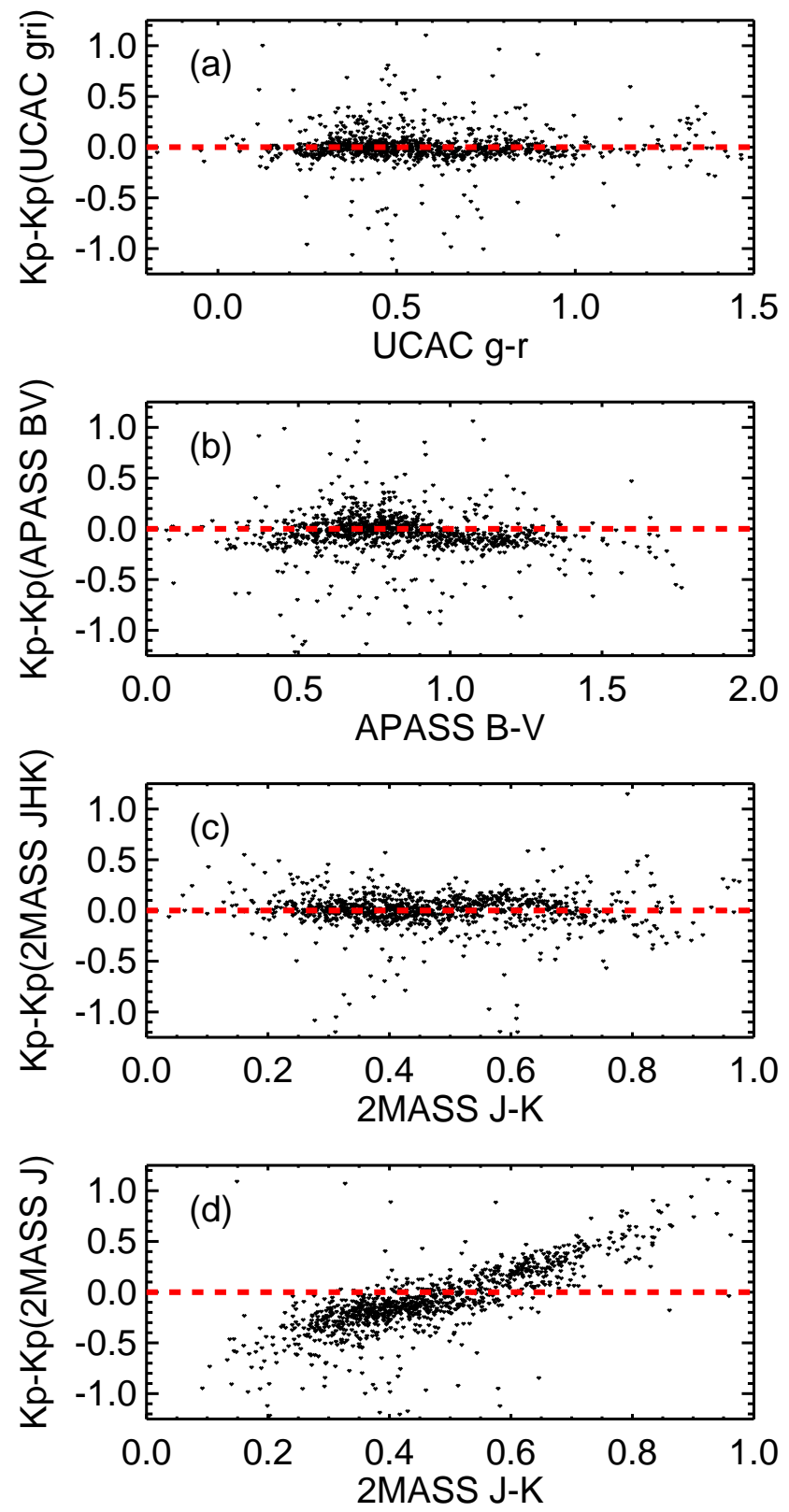

FIG. 2.- Difference between Kepler magnitudes calculated from UCAC gri (panel a), APASS $B V$ (panel b), 2MASS $J H K$ (panel c), and 2MASS $J$ (panel d) as a function of color for 5000 random targets in the original Kepler field.

2012). The following section describes this procedure for $\mathrm{C} 0$, an engineering run for which the this reconciliation was particularly important since target selection began before an EPIC was generated. The procedures described below are also used for later campaigns, and hence apply to most proposers who submitted targets without an existing EPIC ID.

For C0, EPIC IDs were assigned to the 7748 targets approved for flight during the proposal review and target management process. This set of observed targets is referred to below as the "Target Catalog". The only columns populated in this catalog were those provided by the proposers: Kepler magnitude, right ascension, and declination. Subsequently, a "Comprehen- 

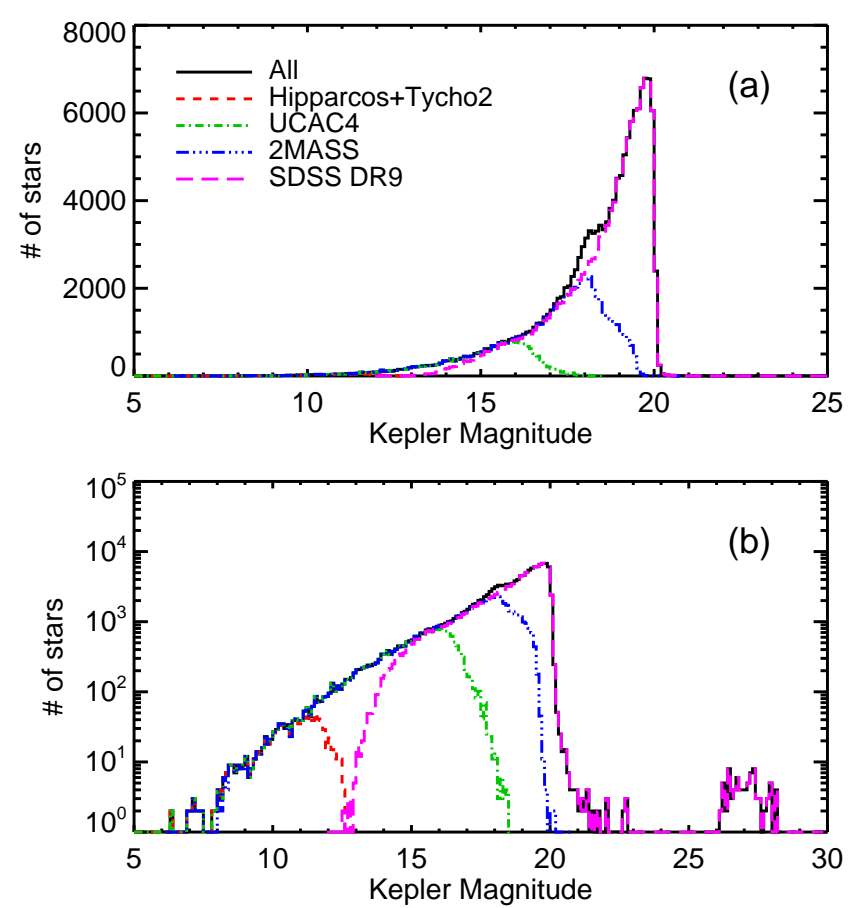

FIG. 3.- Histogram of EPIC Kepler magnitudes for sources in a $\approx 80$ square degree field in C1 covered by both SDSS and 2MASS on a linear scale (panel a) and logarithmic scale (panel b). Colors show the individual contributions from different catalogs. The completness limit of the EPIC is $K p \approx 19$ for areas covered by SDSS, and $K p \approx 17$ for areas covered only by 2 MASS.

sive Catalog" was created and merged with the Target Catalog. Sources were considered matched if their positions agreed to within $<10$ arcseconds and their Kepler magnitudes agreed to within $<4$ magnitudes. For matched sources, the Target Catalog was given precedence over the Comprehensive Catalog, therefore trusting the positions and magnitudes provided by successful proposers. This means that all $\mathrm{C} 0$ targets do not contain cross-matched identifiers or broadband magnitudes as described in Section 2.1 in the EPIC10, and that the listed Kepler magnitudes were calculated differently than described in Section 3 .

A total of 137 sources in the C0 Target Catalog had no match in the Comprehensive Catalog for C0, and underwent closer inspection. For these unmatched sources, the following procedure was adopted:

(1) If $K p>14$ (80 targets), unmatched Target Catalog sources were included with no change because the Comprehensive Catalog is significantly incomplete at faint magnitudes.

(2) If $K p<14$ (11 targets) and the Target Catalog had a duplicate source, then the source that best agreed with the Comprehensive Catalog was included and the nearby duplicate was forced to $K p=30$, thereby declaring it an artifact. An example of this situation is shown in Figure 4 a. In

10 Following community advocacy, the C0 EPIC cross-matches have been made available as a separate delivery at MAST (http://archive.stsci.edu/k2/catalogs.html). We caution that it cannot be guaranteed that the cross-matched information corresponds to the intended targets proposed by the community. such cases, the K2 photometry pipeline is expected to produce valid photometry for the first source, but will only export calibrated pixel-level data for the second. Consequently, proposers who submitted poor source coordinates might find more completely processed data under an alternate EPIC ID.

(3) If $K p<14$, the Target Catalog has no duplicate source, and:

(a) $K p>12$ (23 targets), then the magnitude of the source was forced to $K p=30$. Several examples are shown in Figure $4 \mathrm{~b}$. The rationale is that the Comprehensive Catalog is expected to be complete at these magnitudes, so unmatched sources are likely artifacts.

(b) $K p<12$ (2 targets), and a corresponding source was found in the Comprehensive Catalog within a distance $<30^{\prime \prime}$, then the Target Catalog source was replaced by its match in the Comprehensive Catalog. An example is shown in Figure 4k. The rationale is that for these bright sources uniqueness is less of an issue, so the search space was expanded to obtain matches that led to more reliable parameters. For $\mathrm{C} 0$ the aperture masks included a large halo of 10 pixels (40") in all directions, and hence the requisite pixels for the intended target were likely collected.

(c) $K p<12$ (14 targets), and no corresponding source was found in the Comprehensive Catalog within $<30^{\prime \prime}$, then the magnitude of the source was forced to Kepmag $=30$. The rationale is that larger offsets probably push the proposed targets outside their aperture masks even if the intended sources could be correctly identified in the Comprehensive Catalog.

(4) The remaining (7) targets were included because they were judged to be plausible omissions or magnitude mismatches in the Comprehensive Catalog based on visual checks with POSS-II images.

Details on C0 Target Catalog overrides can be found in the online EPIC documentation 11 . Similar corrections as described in this section were also applied to proposed targets without a cross-matched EPIC ID after C0.

\subsection{Duplicate Sources for Bright Stars with Infrared Excess and Faint SDSS Stars}

Matches between Hipparcos and 2MASS are based on position and apparent magnitudes, the latter being estimated through empirical conversions from 2MASS bands to $V$. For stars with strong infrared excess (such as young stars with debris disks), the conversion from $J H K$ to $V$ can produce wrong $V$-band estimates, causing the EPIC code to erroneously generate two bright sources since the apparent magnitude match fails. An example is T Tauri, which was catalogued with two EPIC IDs (EPIC 210777988 and EPIC 210777987). In general, such errors should be rare since such optical-infrared magnitude matches are restricted to Hipparcos and Tycho.

11 http://archive.stsci.edu/k2/epic.pdf 

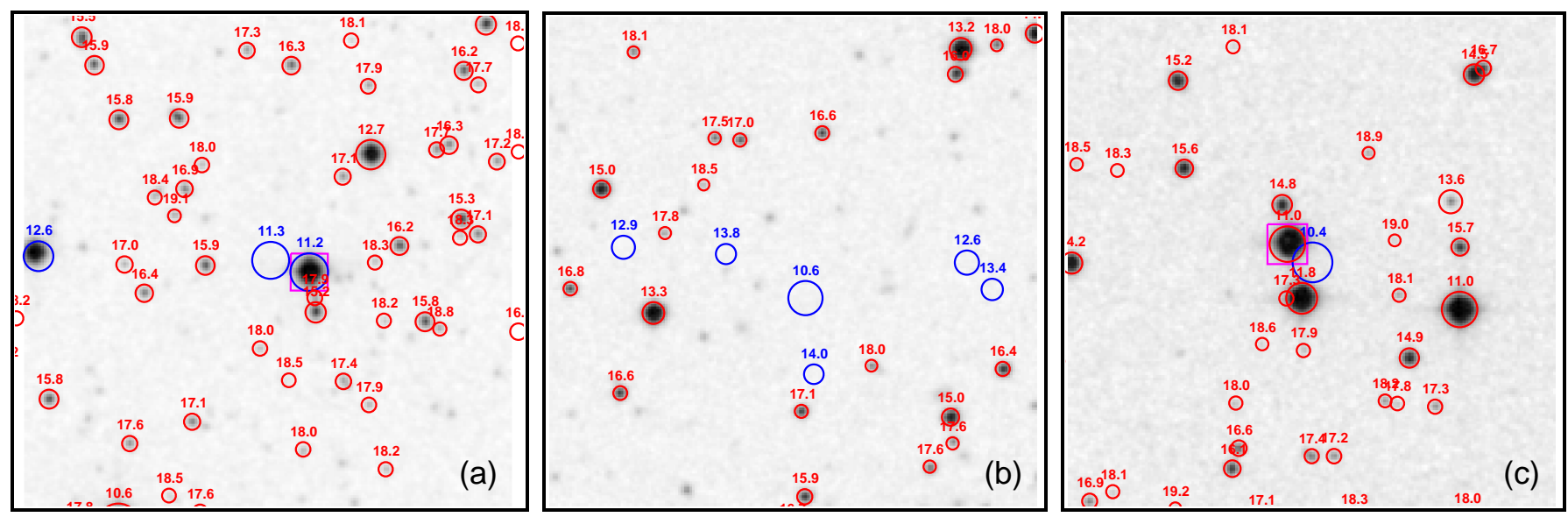

FIG. 4.- POSS-2 images in the C0 field with EPIC sources from the Comprehensive Catalog overlaid (red circles). Each image spans $3 \times 3$ arcminutes and circle sizes scale with Kepler magnitude, which is given next to each source. Each panel demonstrates cases of reconciling sources provided by the community (Target Catalog, blue circles) with the EPIC: duplicate sources in the Target Catalog (panel a), erroneous sources in the Target Catalog (panel b), and source merging between the Target and Comprehensive Catalog (panel c). Magenta squares in panels a and c show the adopted source.

Similar to the Hipparcos-2MASS cross-match, sources between 2MASS and SDSS are matched using positions and apparent magnitudes by applying transformations from $J H K$ to Sloan $g$. If no 2MASS $H K$ photometry with quality flag better than $\mathrm{C}$ is available, this magnitude match is dropped and only a position match is required. Due to a coding error this criterion was not correctly applied for $\mathrm{C} 0-\mathrm{C} 7$, and hence a fraction of faint sources may have erroneously duplicated EPIC sources generated from the same 2MASS and SDSS source.

\subsection{Missing NOMAD Proper Motions}

Following community advocacy, starting from C2 proper motions in the EPIC were supplemented by values provided in the NOMAD1 catalog (Zacharias et al. 2005). These overrides were intended to be only applied for sources that do not have proper motions from the other input catalogs (i.e. Tycho, UCAC, and SDSS). Due to a coding error this criterion was not correctly applied in $\mathrm{C} 3-\mathrm{C} 7$, and consequently for some Tycho/UCAC/SDSS stars proper motions were replaced with NOMAD values, or were left blank although NOMAD proper motions exist. Identifiers were in all cases propagated correctly, and hence all sources with a NOMAD identifier have their proper motion values taken from the NOMAD catalog.

\subsection{Missing Sources due to Quality Flag Cuts}

In rare cases the quality cuts applied for 2MASS photometry may remove genuine infrared bright sources with no optical counterparts. One example for this case is QQ Tau, which has no $B V$ gri photometry in UCAC4 and a $J$-band E quality flag. Since 2MASS $H K$ are not sufficient to calculate a Kepler magnitude the source was dropped from the EPIC, despite its bright $J$-band magnitude. QQ Tau was consequently added to the EPIC after being successfully proposed for observations in C4, following the procedures outlined in Section 4.1.

\subsection{Regions of Incompleteness}

For $\mathrm{C} 7$ there was no 2MASS data available for a rectangular region about 0.1 degrees in right ascension by 0.3 degrees in declination that is centered near $\mathrm{RA}=287.9^{\circ}$, Dec $=-17.7^{\circ}$ and for a circular region about $0.07^{\circ}$ in diameter that is centered near $\mathrm{RA}=285.3^{\circ}$, Dec $=-22.7^{\circ}$. While the latter is almost certainly due to masking associated with the 8.2 magnitude star at the center of the region, the former is not understood and sky images do show bright sources in that area. The result is that the EPIC is much less complete in these two regions than in the remainder of the $\mathrm{C} 7$ field of view. These regions are relatively small compared to the region caused by $\alpha$ Sco in the C2 (see Figure 1). We emphasize that regions of incompleteness likely exist for most campaigns, and hence users should be aware that certain photometric bands in the EPIC may be affected by this.

\subsection{Reduced Completeness for C9}

Because C9 is near the galactic plane and includes portions of the galactic bulge, the stellar density is at least a factor of ten higher than for other campaigns. Consequently, the 2MASS input catalog was truncated at $J<14$ in order to limit the number of catalog entries for C9 to 7.9 million for computational reasons (see Table 2). Because UCAC4 is typically complete to $K p \approx 15$, the EPIC is likely complete to about this magnitude, but the resulting estimates are less accurate due to large reddening in this field. Additionally, due to differential reddening between optical and infrared magnitudes, $K p$ values derived from $J H K$ will be underestimated (too bright) compared to $K p$ values derived from optical bands. Fortunately, these issues are expected to have little impact on the aperture sizes used for data collection, particularly since most pixels collected during C9 will reside in large super apertures to facilitate a microlensing experiment (Henderson et al. 2015). Due to code improvements comprehensive catalogs in crowded fields following C9 are no longer magnitude limited, as can be seen by the $\mathrm{C} 11 \mathrm{EPIC}$ which contains $\approx 17$ million sources.

\subsection{Systematic Offsets in Kepler Magnitudes}

Early K2 campaigns revealed that EPIC $K p$ values derived from 2MASS photometry (Kepflag='JHK' or Kepflag='J', see Section [3) are systematically too low 
(too bright) by about one magnitude compared to flux measurements from the K2 data 12 . Further analysis showed that the majority of the affected targets are faint $(K p>14)$ and red $(J-K>0.8)$, consistent with $\mathrm{M}$ dwarfs. We were unable to reproduce this offset by comparing $K p$ calculated from $J H K$ with $K p$ values in the KIC, or by comparing $K p$ calculated from $J H K$ with $K p$ values calculated from APASS gri in C4. This indicates that the offset is likely due to a discrepancy in the definition of $K p$ compared to actual flux measurements by the spacecraft for red stars, which has so far went unnoticed due to relatively small number of red stars targeted in the Kepler field. However, because the offset causes the K2 apertures to be too large, this should not significantly affect science investigations for these targets.

A second population of stars in C4 showed $K p$ values which are systematically too high (too faint) compared to actual flux measurements. In contrast to targets with underestimated magnitudes, this sample appeared localized in certain regions of the K2 field of view. Further analysis showed that the erroneous $K p$ values are caused by $i$-band values with large uncertainties which are systematically too high, originally adopted from APASS. Future catalogs will mitigate this problem by disregarding photometry with large uncertainties when calculating Kepler magnitudes.

\section{STELLAR CHARACTERIZATION}

Unlike the KIC for the Kepler Mission, the EPIC does not include stellar properties for each source. Consequently, target selection for K2 has relied mostly on color and proper motion cuts. The most comprehensive source for stellar classifications of K2 targets to date is the TESS target selection catalog (Stassun et al. 2014). As of yet, however, this catalog only includes luminosity classes and effective temperatures, and does not take into account reddening which can lead to biases in the effective temperature scale. In this section, we derive a full set of stellar properties (temperatures, surface gravities, metallicities, radii, masses, densities, distances, and extinction) for K2 targets to investigate the target populations, and provide the community with a resource for a first-look characterization of K2 targets. We do not classify all EPIC sources due to computational reasons and to keep the community target selection process free of biases which are inherently present for model-based stellar parameter inference.

\subsection{Apparent Magnitude Distribution of K2 Targets}

Figure 5 shows the EPIC Kepler magnitude distribution for 156,456 K2 targets selected for observations in $\mathrm{C} 1-\mathrm{C} 8$, grouped into campaigns with similar heights above the galactic plane. Most campaigns have a bimodal distribution as a result of programs targeting bright FGK dwarfs and red giants for transiting planet searches and galactic archeology (e.g. Vanderburg et al. 2015; (Stello et al. 2015), while M dwarf exoplanet surveys (e.g. Crossfield et al. 2015) typically propose fainter targets. The distributions show that fields which are high above the galactic plane $(\mathrm{C} 1, \mathrm{C} 3, \mathrm{C} 6$, and $\mathrm{C} 8)$ tend to include fainter targets than those close to the plane $(\mathrm{C} 2$,

\footnotetext{
12 http://keplerscience.arc.nasa.gov/K2/C4drn.shtm
}
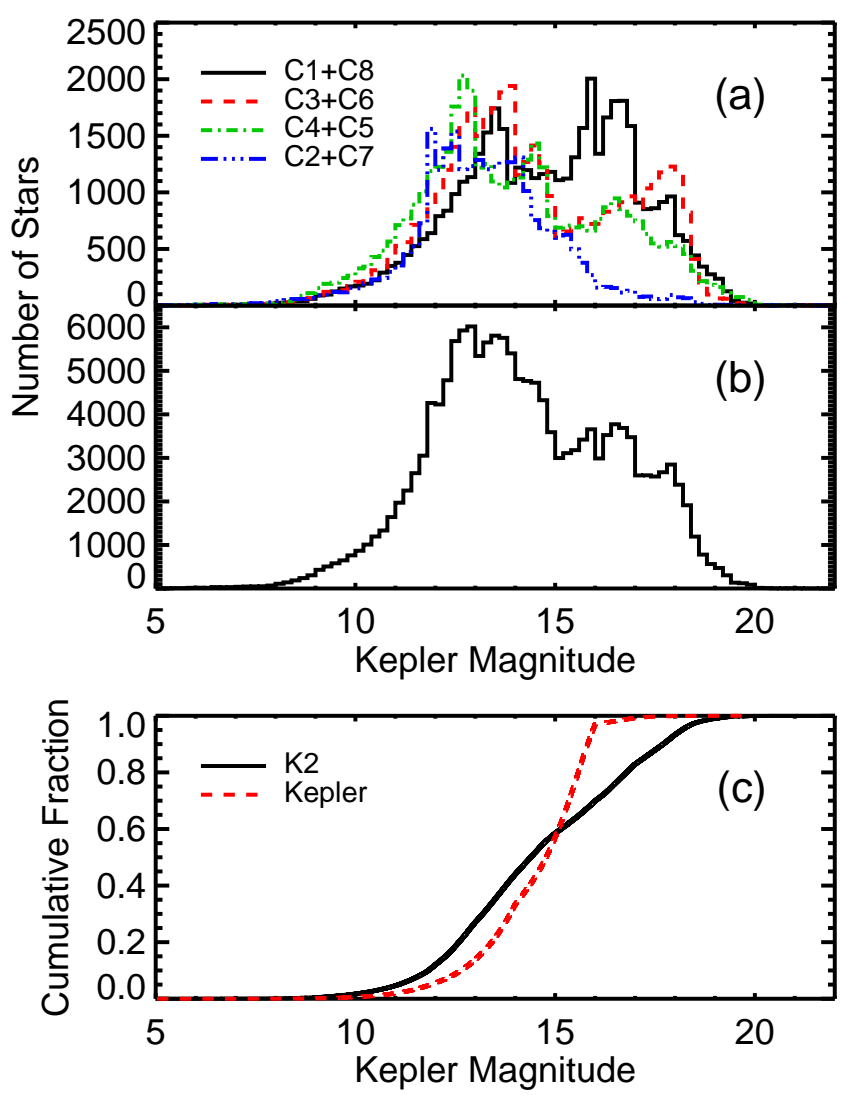

FIG. 5.- Panel (a): EPIC Kepler magnitude distribution for 156,456 K2 targets in C1-C8, split into campaigns with similar heights above the galactic plane: $|b| \approx 57-58^{\circ}(\mathrm{C} 1+\mathrm{C} 8),|b| \approx 50$ $52^{\circ}(\mathrm{C} 3+\mathrm{C} 6),|b| \approx 26-32^{\circ}$ (C4+C5), $|b| \approx 15-19^{\circ}(\mathrm{C} 2+\mathrm{C} 7)$. Panel (b): Kepler magnitude distribution of K2 targets over all campaigns. Panel (c): Cumulative Kepler magnitude distribution of K2 targets (black solid line) compared to the Kepler target sample (red dashed line).

$\mathrm{C} 4$, C5 and $\mathrm{C} 7$ ). Overall, the median apparent magnitude for $\mathrm{K} 2$ targets is $K p \approx 14.0$ and $J \approx 12.2$.

Figure 5c compares the Kepler magnitudes of K2 targets to the distribution of targets in the Kepler field. Interestingly, the median apparent magnitude of $\mathrm{K} 2$ targets is only $\approx 0.6 \mathrm{mag}$ brighter than for Kepler $(K p \approx 14.6)$. However, the K2 target list covers a much broader apparent magnitude distribution, with a larger number of very bright and very faint $(K p>16)$ targets than Kepler.

\subsection{Reduced Proper Motions}

For typical apparent magnitudes of K2 targets the only currently available data are broadband colors and proper motions. Johnson, Sloan or 2MASS filters are sensitive to effective temperatures $\left(T_{\text {eff }}\right)$, but are notoriously insensitive to surface gravities $(\log g)$. For the KIC this problem was alleviated through narrow-band D51 photometry, which provided sensitivity to discern dwarfs from giants. However, D51 photometry or bluer broadband colors with sensitivity to $\log g$ are not available in the all-sky surveys used to construct the EPIC.

An alternative for determining evolutionary states are proper motions. Taking the $J$-band as an example, reduced proper motions are defined as (e.g. 

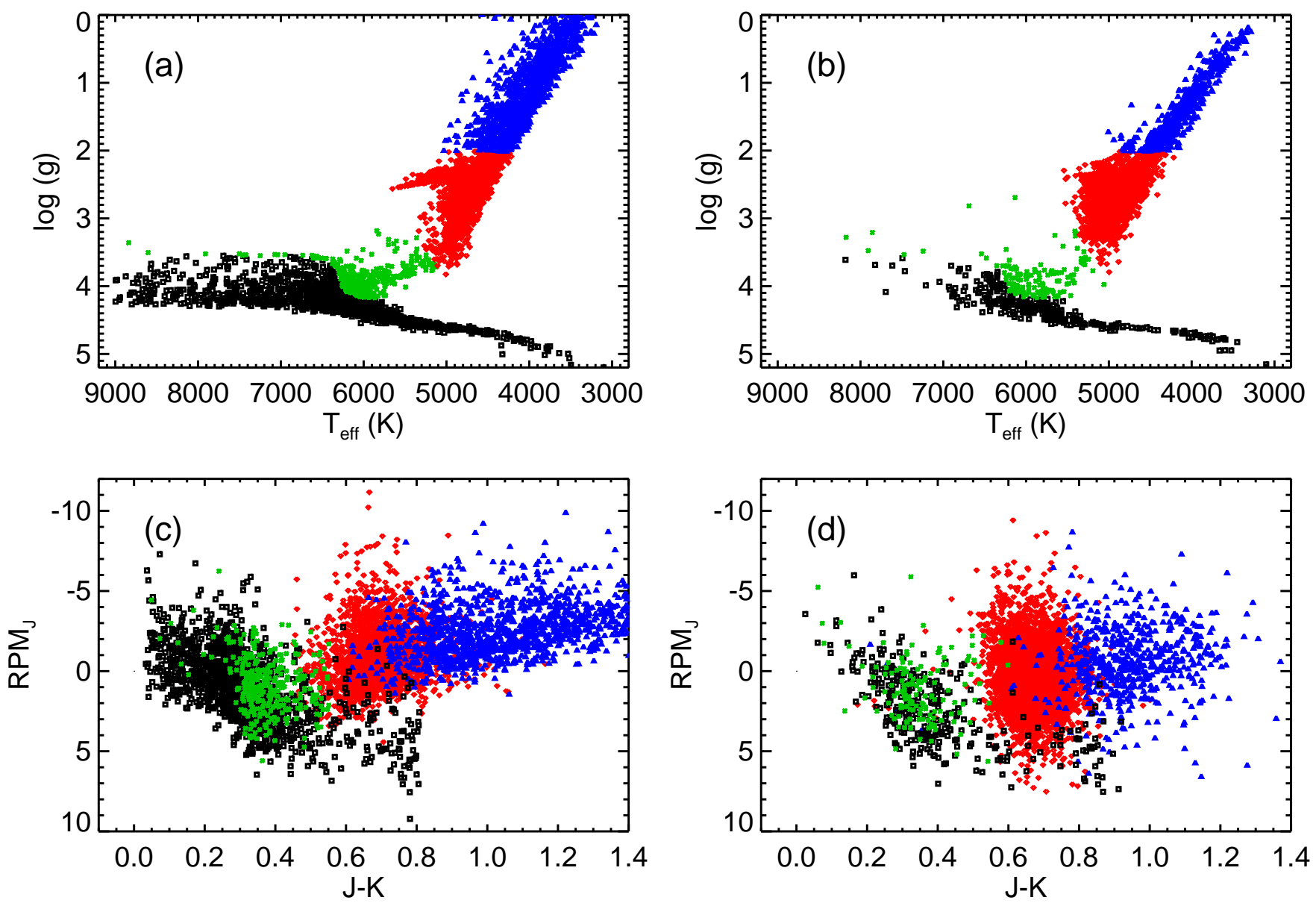

FIG. 6. - $T_{\text {eff }}$ versus $\log g$ for a synthetic population in the Kepler field generated using Galaxia (panel a) with an apparent magnitude distribution which matches a control sample of 17,068 Kepler targets with well determined fundamental properties from asteroseismology and spectroscopy (panel b). Different symbols and colors denote evolutionary states: dwarfs (black squares), subgiants (green asterisks), low-luminosity RGB stars (red diamonds) and high-luminosity RGB stars (blue triangles). Panels (c) and (d) show the same samples as in the top panels but now in a $J$-band reduced proper motion versus $J-K$ color diagram. Only 5000 stars of the full sample are shown for clarity.

\section{Gould \& Morgan 2003):}

$$
\mathrm{RPM}_{\mathrm{J}} \equiv m_{J}+5 \log (\mu)=M_{J}+5 \log \left(\frac{v_{t}}{47.4 \mathrm{~km} \mathrm{~s}^{-1}}\right) \text {. }
$$

Here, $\mathrm{RPM}_{\mathrm{J}}$ is the reduced proper motion in the $\mathrm{J}$-band, $M_{J}$ and $m_{J}$ are the absolute and apparent $J$-band magnitudes, $\mu$ is total proper motion in arcseconds per year and $v_{t}$ is the transverse velocity in $\mathrm{km} \mathrm{s}^{-1}$. Assuming equal transverse motion of all stars, reduced proper motions are equivalent to absolute magnitudes since the apparent motion depends solely on the distance to the observer. In reality, stars show significant dispersion in $v_{t}$, and hence reduced proper motions are only a proxy for absolute magnitudes. Nevertheless, assuming relatively local thin disc populations, proper motions can be effective in characterizating stellar populations.

To test the usefulness of proper motions we first constructed an EPIC for the Kepler field following the procedure described in Section 2.1. We then used Galaxia (Sharma et al. 2011) to simulate a synthetic stellar population in $\mathrm{a} \approx 250$ square degree circle centered on the Kepler field. Galaxia is based on the Besançon model of the galaxy (Robin et al. 2003), with some modifications. It provides model photometry (such as UBV, Sloan and 2MASS), space velocities (U, V, W), and 3D extinction values using a double-exponential disc model with parameters optimized to match the dust maps by Schlegel et al. (1998), along with a correction for high extinction regions as described in Sharma et al. (2014). The isochrones used in Galaxia to predict the stellar properties are from the Padova database (Marigo et al. 2008). For each simulated Galaxia star we calculated proper motions by inverting the equations given by Johnson \& Soderblom (1987).

Figure 6a shows a $T_{\text {eff }}-\log g$ diagram for a subsample of the Galaxia population in the Kepler field (hereafter referred to as the synthetic sample). The subset was randomly drawn to match the apparent $J$-band distribution of a sample of 17,068 Kepler targets with well determined stellar properties from asteroseismology and spectroscopy taken from Huber et al. (2014) (Figure 6b, hereafter referred to as the control sample). Note that the lack of hot stars in Figure $6 \mathrm{~b}$ compared to Figure $6 \mathrm{a}$ is due to the Kepler target selection function. To distinguish evolutionary states, we fitted analytical functions 
to solar-metallicity Parsec models (Bressan et al. 2012) at the terminal age main-sequence and the bottom of the red-giant branch. Based on these fits we classified stars as giants if:

$$
\log (g)< \begin{cases}13.463-0.00191 T_{\text {eff }}, & \text { for } T_{\text {eff }}>5000 \mathrm{~K} \\ 3.9 & \text { otherwise }\end{cases}
$$

while stars were classified as dwarfs if

$$
\log (g)>\frac{1}{4.671} \arctan \left(\frac{T_{\text {eff }}-6300}{-67.172}\right)+3.876 .
$$

Stars were classified as subgiants if they satisfy neither condition (8) nor (9). We note that the above conditions depend on metallicity and are approximate only. However, they are sufficient for qualitatively distinguishing between evolutionary states. For illustrative purposes only we furthermore distinguish high-luminosity from low-luminosity red giants if they have $\log g>2$ (note that strictly speaking this is not a separation of evolutionary states, since stars in the red clump are more evolved than stars at the tip of the red giant branch).

Figures 6re and 6] show the synthetic sample and the control sample in a reduced $J$-band proper motion versus $J-K$ color diagram, with evolutionary states color-coded according to the $T_{\text {eff }}$ and $\log g$ cuts in the top panels. The synthetic sample shows that low-luminosity (red) and high-luminosity (blue) red giants separate cleanly from dwarfs and subgiants, while subgiants (green) mostly overlap with the dwarf population (black). The control sample shows a similar distribution, with the exception of a different color distribution due to the Kepler target selection function. This comparison qualitatively demonstrates that synthetic Galaxia populations reproduce observations and hence can be used to infer evolutionary states that are inaccessible with broadband colors alone.

\subsection{Inference of Stellar Properties using Galaxia}

To quantify Figure 6, we inferred stellar properties for the synthetic and the control sample using JHKgri colors, proper motions, and the Galaxia population. Given a set of observables $x=\{J, J-K, H-K, g-r, r-i, \mu\}$ with Gaussian uncertainties $\sigma_{x}$ and a set of intrinsic parameters $y=$ age, $[\mathrm{Fe} / \mathrm{H}]$, mass, distance $\}$, the posterior probability of the observed star having intrinsic parameters $y$ is given by:

$$
p(y \mid x) \propto p(y) p(x \mid y) \propto p(y) \prod_{i} \exp \left(-\frac{\left(x_{i}-x_{i}(y)\right)^{2}}{2 \sigma_{x, i}^{2}}\right)
$$

Here, $p(y)$ is the prior in the form of an initial mass function, age-metallicity relation, and age-velocity relation which depend on the simulated galactic component. We used Galaxia to generate synthetic stars sampled according to $p(y)$. The posterior probability density function (PDF) was then obtained by summing $p\left(y_{j} \mid x\right)$ for a given intrinsic parameter $j$ (thereby marginalizing over all other model parameters), with a stepsize iterated to yield at least 10 bins within a 1- $\sigma$ confidence interval
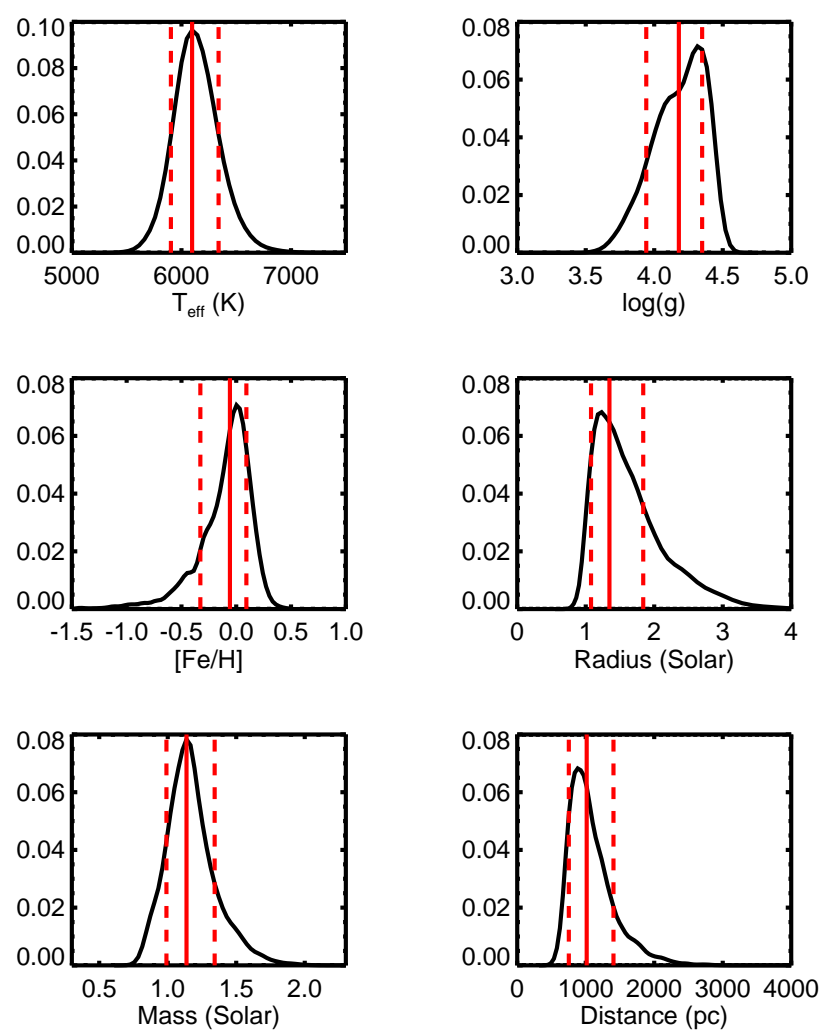

FIG. 7.- Posterior distributions for $T_{\text {eff }}, \log g,[\mathrm{Fe} / \mathrm{H}]$, radius, mass and distance for KIC2438513 $(K p=13.9, J=12.9)$ derived from reduced proper motions, JHKgri photometry, and a Galaxia synthetic population of the Kepler field. Solid and dashed lines show the median and 1- $\sigma$ confidence intervals.

around the median. To avoid discontinuities for parameter spaces with a sparse numbers of models (see also next paragraph) the distributions were smoothed with a Gaussian with a FHWM of 3 bins. Note that the volume in age, mass and metallicity covered by each isochrone point (which is required to take into account different evolutionary speeds, see Casagrande et al. 2011; Serenelli et al. 2013) are taken into account through the sampling process used to generate the Galaxia population. Figure 7 shows example posteriors for a star in the Kepler control sample. For each PDF we record the median (solid red line) and 1- $\sigma$ interval around the median (dashed red lines).

A key advantage of the method is that Galaxia allows to use proper motions as an observable, which is not possible for traditional isochrone grids. A disadvantage is that in a typical synthetic realization by Galaxia bright stars are relatively rare, and hence parameter inference suffers from small number statistics. To overcome this we calculated an oversampled bright population to ensure that the brightest 0.5 mag-wide apparent magnitude bin contains at least 2000 synthetic stars. We note that for the same reason we did not make use of spatial information (i.e. galactic latitude and longitude) of each star, thereby essentially assuming uniform reddening across a given K2/Kepler FOV. Similarly, we undersampled the faint end of the synthetic realization to avoid excessive numbers of synthetic stars. All subpopulations were calculated to overlap by at least $0.5 \mathrm{mag}$, and are used independently for the parameter inference described above 

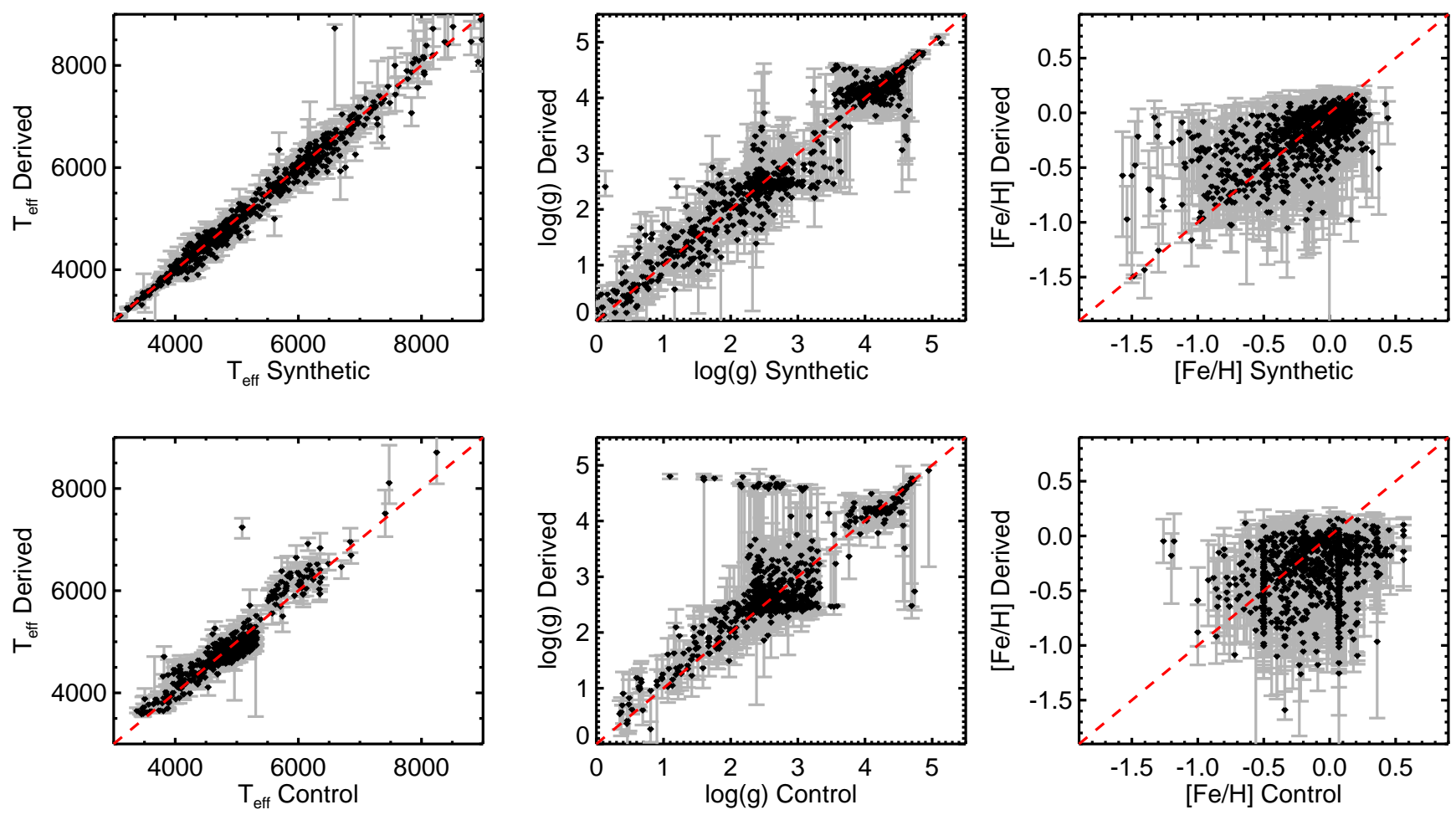

FiG. 8. - Stellar properties derived from proper motions, colors, and a Galaxia synthetic population versus comparison values for $T_{\text {eff }}$ (left panels), $\log g$ (middle panels) and $[\mathrm{Fe} / \mathrm{H}]$ (right panels). Comparison values are taken from a synthetic sample of the Kepler field (top panels, see also Figure 6 $\mathrm{a}$ ) and the Kepler control sample (bottom panels, see also Figure 6 b). Grey lines show 1- $\sigma$ error bars and red-dashed line show the 1:1 relation. Only $5 \%$ of the total sample is shown for clarity.

(i.e., subpopulations are not mixed).

To test the method we perturbed observables from the synthetic sample (Figure 6a) with Gaussian uncertainties typical for the EPIC (0.025 mag for each photometric band, and $2.5 \mathrm{mas} / \mathrm{yr}$ for proper motions), and compared the derived properties to the actual properties of each synthetic star. As a second test, we used actual data from the Kepler control sample (Figure 6b) and compared the results to the stellar properties listed in Huber et al. (2014) (all of which were derived from asteroseismology and/or spectroscopy).

The results are shown in Figure 8 . As expected the synthetic sample performs better than the control sample due to differences between the real data and the synthetic data. $T_{\text {eff }}$ is well recovered, with the control sample showing a slight bias, being on average $\approx 80 \mathrm{~K}$ hotter than the comparison values. We attribute this bias to differences in the color-temperature scale and/or extinction maps between Galaxia and Huber et al. (2014), which mostly adopted $T_{\text {eff }}$ values from Pinsonneault et al. (2012). The right panels of Figure 8 show that the method has very little sensitivity to $[\mathrm{Fe} / \mathrm{H}]$, and essentially recovers the $[\mathrm{Fe} / \mathrm{H}]$ prior from synthetic population. Therefore, metallicities derived in this work are valid in a statistical sense only.

The $\log g$ comparison (middle panels of Figure 8) shows that $1-4 \%$ of giants are misclassified as dwarfs, while 4 $7 \%$ of dwarfs are misclassified as giants. Closer inspection revealed that giants classified as dwarfs are evolved stars with unusually high proper motions, some of which are likely due to catalog errors. The $\log g$ comparison for the Kepler sample shows a slight systematic bias for giants, with derived $\log g$ values being systematically larger. This is consistent with the $T_{\text {eff }}$ bias discussed above, because in the absence of a strong $\log g$ constraint an overestimated temperature on the red-giant branch yields an overestimated $\log g$. As expected from Figure 8, the dwarf-subgiant separation is considerably less successful than for dwarfs and giants: $56-72 \%$ of subgiants are classified as dwarfs, while $9 \%$ of dwarfs are misclassified as subgiants. We emphasize, however, that this is in most cases appropriately captured within the uncertainties: $\approx 79 \%$ (for the control sample) and $\approx 93 \%$ (for the synthetic sample) of all true subgiants would be classified as subgiants within their respective 1- $\sigma$ error bars in $T_{\text {eff }}$ and $\log g$.

We conclude that proper motions and colors from the EPIC combined with a synthetic population generated by Galaxia yield stellar classifications and uncertainties which are suitable for large populations of stars. The overall residual scatter from the tests performed above is $\approx 2-3 \%$ for $T_{\text {eff }} \approx 0.3$ dex in $\log g$ and $\approx 0.3 \mathrm{dex}$ in $[\mathrm{Fe} / \mathrm{H}]$. This is consistent with the performance of the KIC (Bruntt et al. 2010; Kallinger et al. 2010; Verner et al. 2011; Huber et al. 2014), despite the lack of Sloan $z$ and $D 51$ photometry in the EPIC.

\subsection{Stellar Populations in $C 1-C 8$}

To classify K2 targets, we calculated Galaxia populations for each K2 field and applied the method described in the previous section to all EPIC IDs selected for observations. K2 targets were extracted from the K2 web 

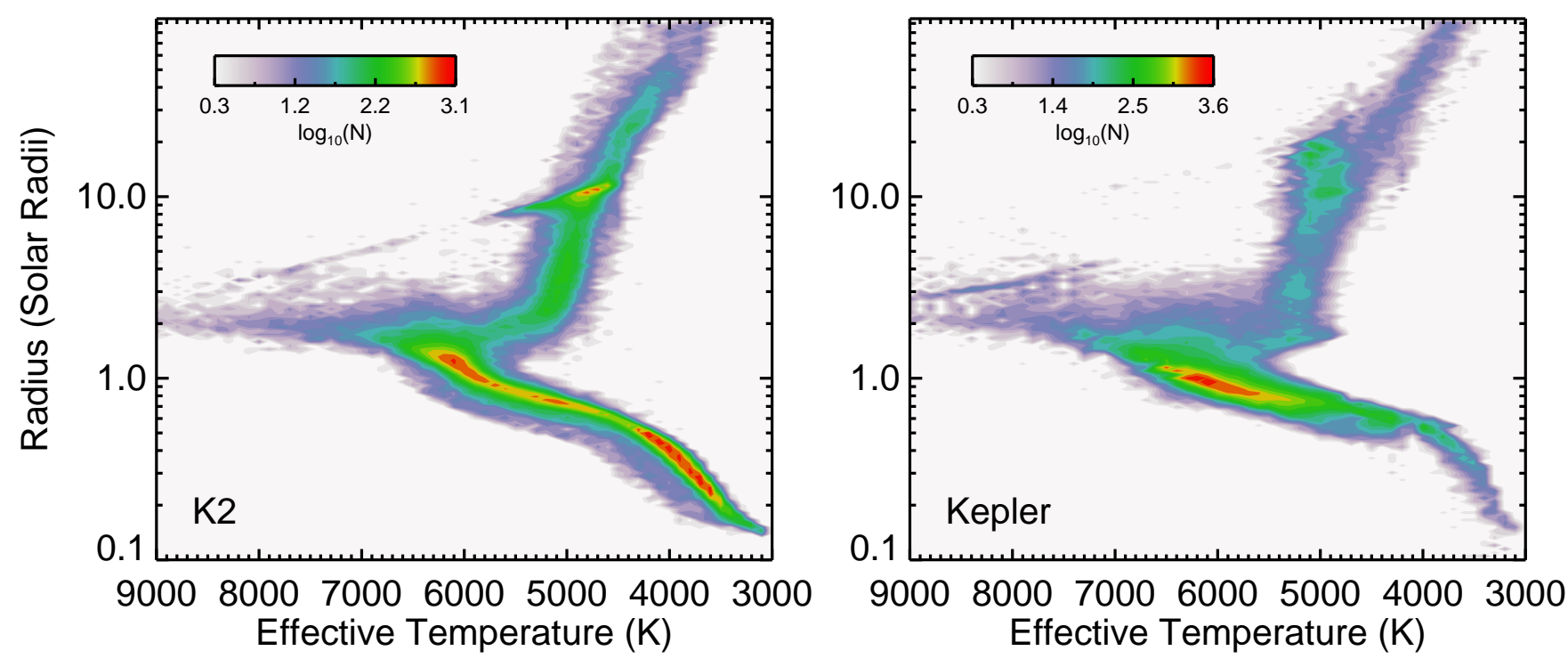

FIG. 9. - Stellar radius versus effective temperature for $138,600 \mathrm{~K} 2$ targets classified in this work (left) and $\approx 190,000 \mathrm{Kepler}$ targets taken from Huber et al. (2014). Colors show the logarithmic number density. Note that differences in the shape of the distribution result mostly from the different isochrones (Padova for K2, Dartmouth for Kepler) which were applied for the classification.

page at the Kepler Science Center 13 . In addition to colors and proper motions, we used parallaxes from Hipparcos van Leeuwen 2007) and spectroscopic information $\left(T_{\text {eff }}\right.$, $\log g$ and $[\mathrm{Fe} / \mathrm{H}]$ ) from the RAVE DR4 (Kordopatis et al. 2013), LAMOST DR1 (Luo et al. 2015) and APOGEE DR12 (Alam et al. 2015) surveys. The spectroscopic sources were matched to the EPIC coordinates with a radius of 3 ". The observables $x$ used to classify a given target were then chosen according to the following priority order:

1) $x=\{J, J-H, H-K, g-r, r-i, \pi\}$ if Hipparcos parallaxes $(\pi)$ were available. If no $J H K$ photometry was available for bright stars with parallaxes, $x=\{V, B-V, \pi\}$ were used. If only a small number of models were available within the uncertainties (e.g. very close M dwarfs) we fitted absolute magnitudes instead of parallaxes and apparent magnitudes, thereby removing the distance constraint. For these cases no $E(B-V)$ values are reported.

2) $x=\left\{J, T_{\text {eff }}, \log g,[\mathrm{Fe} / \mathrm{H}]\right\}$ if a RAVE, LAMOST or APOGEE classification were available. We assumed default uncertainties of $150 \mathrm{~K}$ in $T_{\text {eff }}$, 0.15 dex in $\log g$ and 0.15 dex in $[\mathrm{Fe} / \mathrm{H}]$ for all spectroscopic classifications. We assumed solar metallicity if no metallicity estimate was provided by APOGEE.

3) $x=\{J, J-K, H-K, g-r, r-i, \mu\}$ if proper motions were available.

4) $x=\{J, J-K, H-K, g-r, r-i\}$ if only colors were available.

We assumed a minimum uncertainty of 1 mas/yr for all total proper motions and $0.03 \mathrm{mag}$ for all colors. K2

\footnotetext{
13 http://keplerscience.arc.nasa.gov/index.html
}

targets which are either flagged as extended objects (e.g. galaxies) or have neither valid 2MASS photometry nor a Hipparcos parallax in the EPIC are not classified. For C1-C8 this affects $\approx 12 \%$ of all targets.

Figure 9 shows a $T_{\text {eff-radius number density distribu- }}$ tion of the $138,600 \mathrm{~K} 2$ targets classified in this work compared to $\approx 190,000$ targets in the Kepler field. Stellar properties for the Kepler sample were taken from Huber et al. (2014). The distributions demonstrate the preferential selection of $\mathrm{K} / \mathrm{M}$ dwarfs for $\mathrm{K} 2$, which were underrepresented in the Kepler sample compared to the larger fraction of sun-like dwarfs. K2 also includes a larger relative fraction of red giants compared to dwarfs, a consequence of the $\mathrm{K} 2$ galactic archeology program to map the age and metallicity distributions of stars using asteroseismology (e.g., Miglio et al. 2013; Casagrande et al. 2014; Pinsonneault et al. 2014; Stello et al. 2015). Note that the diagonal "band" near $T_{\text {eff }} \approx 6500-7000 \mathrm{~K}$ and $R \approx 4-7 R_{\odot}$ is due to the sparseness of metal-poor horizontal branch models in Galaxia.

Figure 9 shows systematic differences between the K2 and Kepler samples due to the use of different models: Galaxia adopts Padova isochrones (Girardi et al. 2000; Marigo \& Girardi 2007; Marigo et al. 2008) while the Kepler stellar properties catalog is based on isochrones from the Dartmouth Stellar Evolution Program (Dotter et al. 2008). Specifically, radii for $M$ dwarfs at fixed $T_{\text {eff }}$ are systematically smaller in Padova models, which disagrees with empirical measurements from long-baseline interferometry (Boyajian et al.|2012b, 2013) or low-mass eclipsing binary systems (Kraus et al. 2011; Carter et al. 2011). This discrepancy has been improved in the updated Parsec isochrones (Bressan et al. 2012), which however have not yet been implemented in Galaxia. Hence, users should be aware that radii for $\mathrm{M}$ dwarfs derived in this work can be underestimated by up to $\approx 20 \%$. On the other hand, classifications of giants near the red clump are more accurate for the K2 sample 

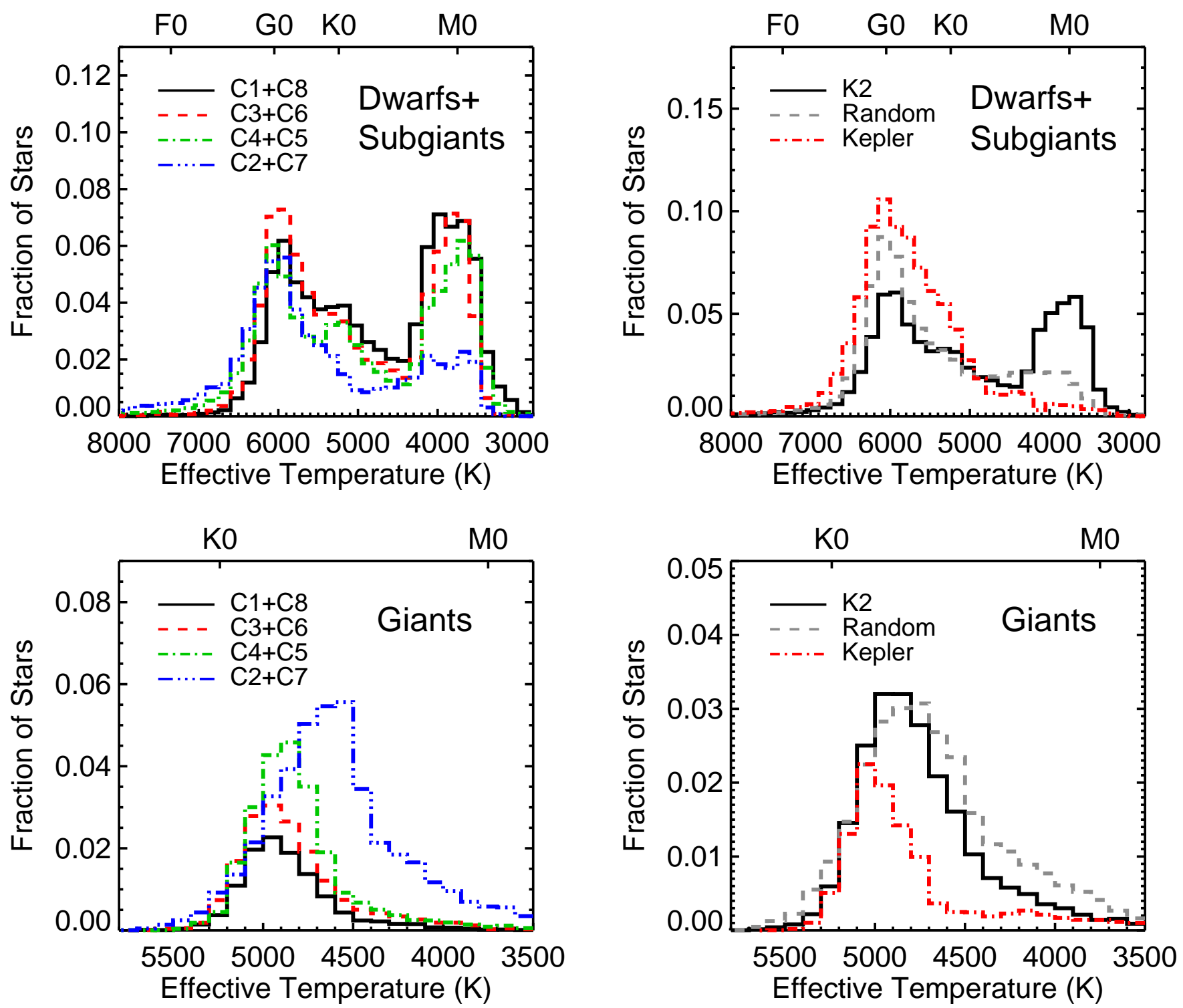

FIG. 10.- Left panels: Effective temperature distributions for K2 targets classified as subgiants and dwarfs (top) and giants (bottom). Targets have been grouped into campaigns with similar heights above the galactic plane: $|b| \approx 57-58^{\circ}(\mathrm{C} 1+\mathrm{C} 8),|b| \approx 50-52^{\circ}(\mathrm{C} 3+\mathrm{C} 6)$, $|b| \approx 26-32^{\circ}$ (C4+C5), $|b| \approx 15-19^{\circ}(\mathrm{C} 2+\mathrm{C} 7)$. The top axis labels show representative spectral types (see also Table 4). Right panels: Same as the left panels but for all K2 campaign targets, the Kepler sample, and a random sample drawn from Galaxia synthetic populations which match the apparent magnitude distribution of K2 targets.

because the Dartmouth models adopted by Huber et al. (2014) did not include He-core burning stars. This can be seen by the correct position of the red clump near $\approx 10 R_{\odot}$ for the K2 sample in Figure 9 .

To quantify the classifications of $\mathrm{C} 1-\mathrm{C} 8$ targets, Figure 10] shows the effective temperature distribution for dwarfs and subgiants (top panels) and giants (bottom panels) subdivided into campaigns at similar heights above the galactic plane (left panels). The luminosity classification was performed using the conditions in Equations (8) and (9). The distributions show several interesting differences in the target populations between different campaigns. For example, campaigns which are close to the galactic plane $(\mathrm{C} 2$ and $\mathrm{C} 7$ ) show a much lower fraction of $\mathrm{M}$ dwarfs but a larger relative fraction of hot dwarfs and cool giants. We suspect that this is partially due to the increased number of giants in a magnitude limited sample in the galactic plane as well as the effect of higher interstellar reddening, the combination of which led to a preferential selection of cool giants and hot dwarfs compared to cool dwarfs. The effect can also be seen in the temperature distribution of giant stars (bottom left panel): since more red stars are classified as giants in lower latitude fields, the average $T_{\text {eff }}$ for giants is cooler than in higher latitude fields. We caution, however, that uncertainties in the reddening model adopted in Galaxia may also contribute to this effect. The top left panel furthermore shows a slight overabundance of $\mathrm{K}$ dwarfs and subgiants with $T_{\text {eff }} \approx 5200 \mathrm{~K}$ in fields at high latitudes, which can also tentatively be seen in Figure 9. The reason for this overabundance is unknown, but is likely due to a systematic misclassification of gi- 
ants as dwarfs rather than a preferential target selection by the community.

The right panels of Figure 10 compare the $T_{\text {eff }}$ distribution of K2 targets to the Kepler sample (Huber et al. 2014). Additionally, we show a randomly drawn sample from the synthetic populations generated by Galaxia, which for each campaign reproduces the apparent magnitude distribution of the actual K2 target list. As expected, for dwarfs and subgiants there is a clear overabundance of $\mathrm{K} / \mathrm{M}$ dwarfs for $\mathrm{K} 2$ compared to Kepler and the random sample, while the Kepler sample is biased towards sun-like dwarfs and against cool dwarfs. For giants the distribution is largely consistent with a random selection, in agreement with the target selection function of the galactic archeology program (which uses a $J-K>0.5$ color cut). Overall, the distributions are consistent with the primary K2 science programs.

Table 4 summarizes the spectral types of K2 targets in $\mathrm{C} 1-\mathrm{C} 8$ presented here. Overall, the most common $\mathrm{K} 2$ targets are $\mathrm{K}-\mathrm{M}$ dwarfs $(\approx 41 \%$ of all classified targets) followed by $\mathrm{F}-\mathrm{G}$ dwarfs $(\approx 36 \%)$ and red giants $(\approx 21 \%)$. We emphasize that there is considerable variation in these classifications from campaign to campaign.

\subsection{K2 Exoplanet Host Stars}

Figure 11 compares effective temperatures (panel a) and stellar radii (panel b) from this work compared to values published in K2 planet discovery papers. Most literature values are taken from Montet et al. (2015), who derived posteriors by comparing broadband colors to Dartmouth isochrones using Nested Sampling (Morton 2015). Additionally we have added K221 (Petigura et al. 2015), K2-22 (Sanchis-Oieda et al. 2015), as well as host stars from Sinukoff et al. (2015), with stellar properties derived from high-resolution spectra using Specmatch (Petigura 2015). We note that K2-2 (Vanderburg et al. 2015) was not included since it was observed during the K2 engineering run, which is not covered in our catalog.

Figure 11] shows good agreement for $T_{\text {eff, }}$ with an offset of $\approx 1 \%$ and a residual scatter of $\approx 3 \%$, which is consistent within the reported uncertainties. Figure $11 \mathrm{~b}$ shows that radii for stars below $\lesssim 0.6 R_{\odot}$ are systematically lower in our sample, which is consistent with the systematic offset between Padova and Dartmouth isochrones discussed in the previous Section. Radii for $\mathrm{G}-\mathrm{K}$ type dwarfs are broadly in agreement.

Several K2 planet hosts show significant radius differences which cannot be explained by isochrone systematics. K2-11 (EPIC 201596316) yielded a bimodal dwarf-giant classification in Montet et al. (2015) $\left(T_{\text {eff }}=\right.$ $\left.5433_{-144}^{+49} \mathrm{~K}, R=5.15_{-4.39}^{+0.20} R_{\odot}\right)$, whereas our results (based on a spectroscopic classification from LAMOST) favor a $\mathrm{K}$ dwarf $\left(T_{\text {eff }}=5240_{-160}^{+160} \mathrm{~K}, R=0.82_{-0.05}^{+0.05} R_{\odot}\right)$, which would firmly place the planet in Earth-sized regime $\left(\approx 1.2 R_{\oplus}\right)$. Conversely, K2-6 (EPIC 201384232) was classified as a sun-like dwarf by Montet et al. (2015) $\left(T_{\text {eff }}=5850_{-98}^{+79} \mathrm{~K}, R=0.96_{-0.09}^{+0.14} R_{\odot}\right)$, consistent with high-resolution spectroscopy by Vanderburg et al. (2016), while our results (based on a spectroscopic classification from RAVE) favor a K-subgiant $\left(T_{\text {eff }}=\right.$ $\left.5370_{-213}^{+255} \mathrm{~K}, R=2.32_{-0.23}^{+0.42} R_{\odot}\right)$. Our results for $\mathrm{K} 2-9$ (EPIC 201465501) favor a hotter and larger late-K dwarf
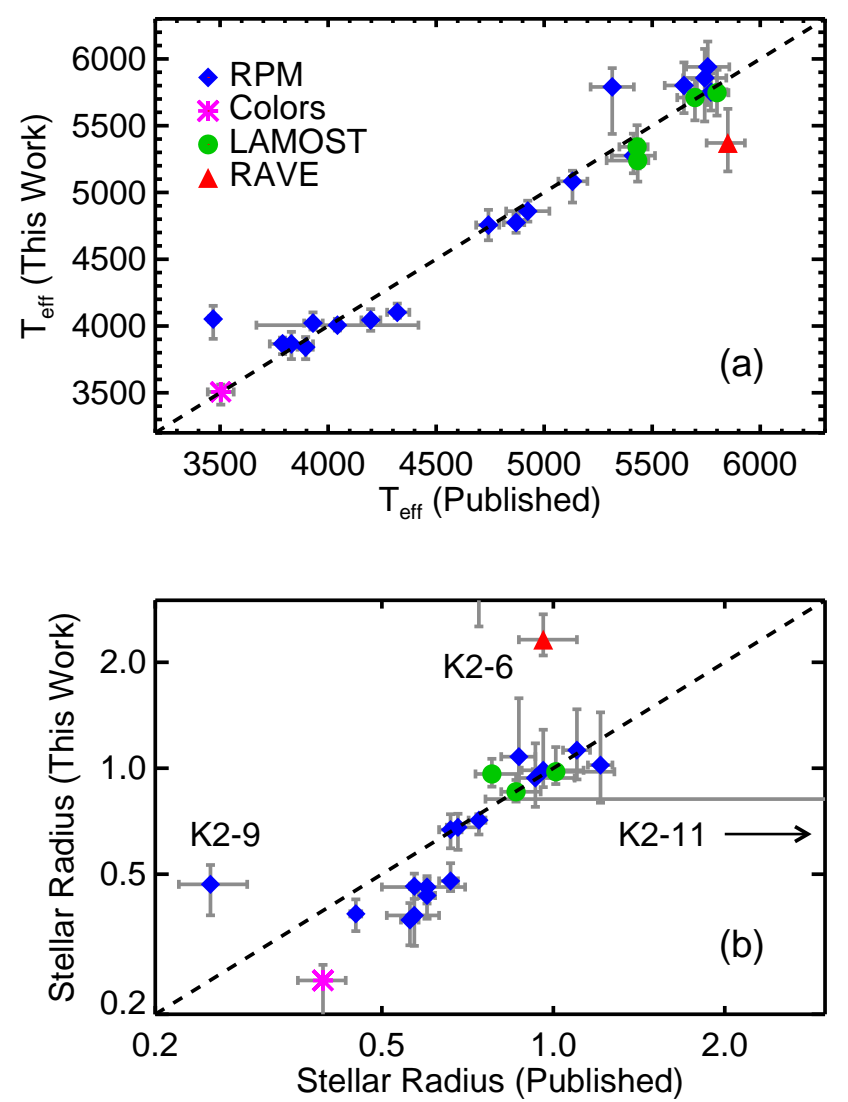

FIG. 11. - Comparison of effective temperatures (panel a) and radii (panel b) of $\mathrm{K} 2$ planet host stars derived in this work versus values published in the literature. Different colors and symbols indicate the classification method used. Three host stars which are discussed in more detail in the text are marked.

$\left(T_{\text {eff }}=4052_{-148}^{+99} \mathrm{~K}, R=0.47_{-0.08}^{+0.06} R_{\odot}\right)$ compared to the mid-M classification by Montet et al. (2015) $\left(T_{\text {eff }}=\right.$ $\left.3468_{-19}^{+20} \mathrm{~K}, R=0.25_{-0.03}^{+0.04} R_{\odot}\right)$, which was later confirmed spectroscopically by Schlieder et al. (2016). Closer inspection showed that while $J-K$ is compatible with a mid-M dwarf, $H-K$ indicates a hotter solution which combined with the fact that very red $J=12.5$ mag dwarfs should be rare skews our posteriors towards $T_{\text {eff }} \approx$ 4000 K. Finally, EPIC 201713348 and EPIC 203826436 are classified as evolved stars $\left(R>3 R_{\odot}\right)$ while the spectroscopic classifications by Sinukoff et al. (2015) imply late-type dwarfs $\left(R<1 R_{\odot}\right)$. We suspect that the discrepancies are due to underestimated uncertainties in the proper motions adopted in the EPIC (which are consistent with evolved stars), and note that the spectroscopic solutions should be preferred.

Finally, we compared $T_{\text {eff }}$ and $\log g$ values for 36 planet-candidate hosts with the catalog by Vanderburg et al. (2016), who presented high-resolution spectra analyzed using SPC (Buchhave et al. 2012, 2014). We find satisfactory agreement with residual scatter of $\approx 180 \mathrm{~K}$ in $T_{\text {eff }}$ and $\approx 0.2$ dex for $\log g$. In addition to the two hosts discussed above, seven stars are classified as evolved $(\log g \approx 2.5-4.0)$ in the EPIC while they are classified as main-sequence stars $(\log g>4.3)$ based on SPC. Three of these hosts (EPIC 201384232, EPIC 205944181 and EPIC 205950854) are based 
TABLE 4

Stellar Classifications of K2 TARgets

\begin{tabular}{|c|c|c|c|c|c|c|c|c|c|c|c|}
\hline Spectral Type & $\mathrm{C} 1$ & $\mathrm{C} 2$ & C3 & $\mathrm{C} 4$ & $\mathrm{C} 5$ & $\mathrm{C} 6$ & $\mathrm{C} 7$ & C8 & $\mathrm{C} 1-\mathrm{C} 8$ & Random & Kepler \\
\hline \multicolumn{12}{|c|}{ Dwarfs \& Subgiants (\%) } \\
\hline A & 0.1 & 2.7 & 0.5 & 1.9 & 0.5 & 0.4 & 3.4 & 0.2 & 1.0 & 1.0 & 1.9 \\
\hline $\mathrm{F}$ & 5.9 & 22.0 & 12.6 & 18.3 & 12.9 & 11.6 & 12.0 & 9.8 & 12.7 & 19.9 & 30.7 \\
\hline $\mathrm{G}$ & 14.5 & 17.3 & 25.7 & 17.8 & 20.4 & 28.3 & 20.6 & 35.0 & 23.0 & 27.6 & 40.4 \\
\hline $\mathrm{K}$ & 44.5 & 9.9 & 34.0 & 22.5 & 29.7 & 30.4 & 17.0 & 33.4 & 28.9 & 21.1 & 14.4 \\
\hline M & 22.5 & 4.1 & 11.4 & 16.2 & 13.3 & 11.4 & 5.6 & 11.1 & 12.4 & 3.1 & 1.4 \\
\hline \multicolumn{12}{|c|}{ Giants (\%) } \\
\hline G & 0.3 & 0.9 & 0.5 & 0.6 & 0.3 & 0.8 & 1.9 & 0.5 & 0.7 & 1.4 & 0.4 \\
\hline K & 12.1 & 41.4 & 15.3 & 22.2 & 22.7 & 17.1 & 37.4 & 9.9 & 20.9 & 24.9 & 10.4 \\
\hline M & 0.0 & 1.6 & 0.1 & 0.4 & 0.2 & 0.1 & 2.2 & 0.1 & 0.5 & 0.9 & 0.6 \\
\hline \multicolumn{12}{|c|}{ Total Classified (\%) } \\
\hline & 83.9 & 98.0 & 83.1 & 95.1 & 87.5 & 86.5 & 94.7 & 80.2 & 87.5 & - & - \\
\hline
\end{tabular}

Notes: Luminosity classifications were assigned from the derived $T_{\text {eff }}$ and $\log g$ using the criterium in Equation 8 Spectral types were assigned using the following temperature ranges: > $7350 \mathrm{~K}(\mathrm{~A}), 6050-7350 \mathrm{~K}(\mathrm{~F}), 5240-6050 \mathrm{~K}(\mathrm{G}), 3750-5240 \mathrm{~K}(\mathrm{~K})$ and $<3750 \mathrm{~K}$ $(\mathrm{M})$. The second to last column lists classifications for a random sample drawn from the Galaxia simulations with the same apparent magnitude distribution as the K2 sample. The classifications of Kepler targets in the last column is based on Huber et al. (2014).

on RAVE spectroscopic classifications, while the remaining four (EPIC 204890128, EPIC 206011496, EPIC 206026904 and EPIC 206114630) were classified using proper motions. While the catalog uncertainties should in most cases capture such misclassifications, these differences illustrate that stellar properties in the EPIC are no substitute for follow-up observations to precisely characterize planets discovered by K2.

\subsection{K2 Asteroseismic Giants}

Stellar oscillations can be used to precisely measure fundamental properties in red giants with long-cadence data (e.g. Kallinger et al. 2010) and hence test derived stellar properties in the catalog. Since the majority of stars with $\log g \approx 2-3.4$ and $K p<15$ should show detectable oscillations with K2 (Stello et al. 2015), asteroseismic analyses are also efficient to discern luminosity classes of Kepler and K2 targets (Huber et al. 2013).

We have performed an asteroseismic analysis of $678 \mathrm{~K} 2$ targets in C5 which have been targeted by the SAGA survey Casagrande et al. 2014). We used PDC-SAP light curves and applied iterative 4- $\sigma$ clipping as well as a high-pass filter with a 2-day width to prepare the data for the asteroseismic analysis. A second, independent analysis used gap-filling to minimize the effects of the 6 hour repointing cycle (Stello et al. 2015). Both datasets were searched for oscillations using the method by Huber et al. (2009).

Out of the 678 targets that were analyzed, 612 have been classified in the EPIC. Figure 12 a shows a $\log g-T_{\text {eff }}$ diagram using the EPIC classifications for all 612 stars (black symbols) and the subset of 70 stars for which oscillations were detected (red symbols). Of the stars with detected oscillations two were misclassified as dwarfs in the EPIC, confirming the low misclassification rate derived in Section 5.3. We note that both misclassified giants have uncertainties that place them on the giant branch within 1- $\sigma$. Twenty seven stars are predicted to be red giants by the EPIC, but do not show oscillations. Inspection of the K2 data showed that about 4 stars oscillate with periods too long to be resolved with a single K2 campaign (high-luminosity giants), while 18 stars are low $\mathrm{S} / \mathrm{N}$ detections or targets for which the light curves
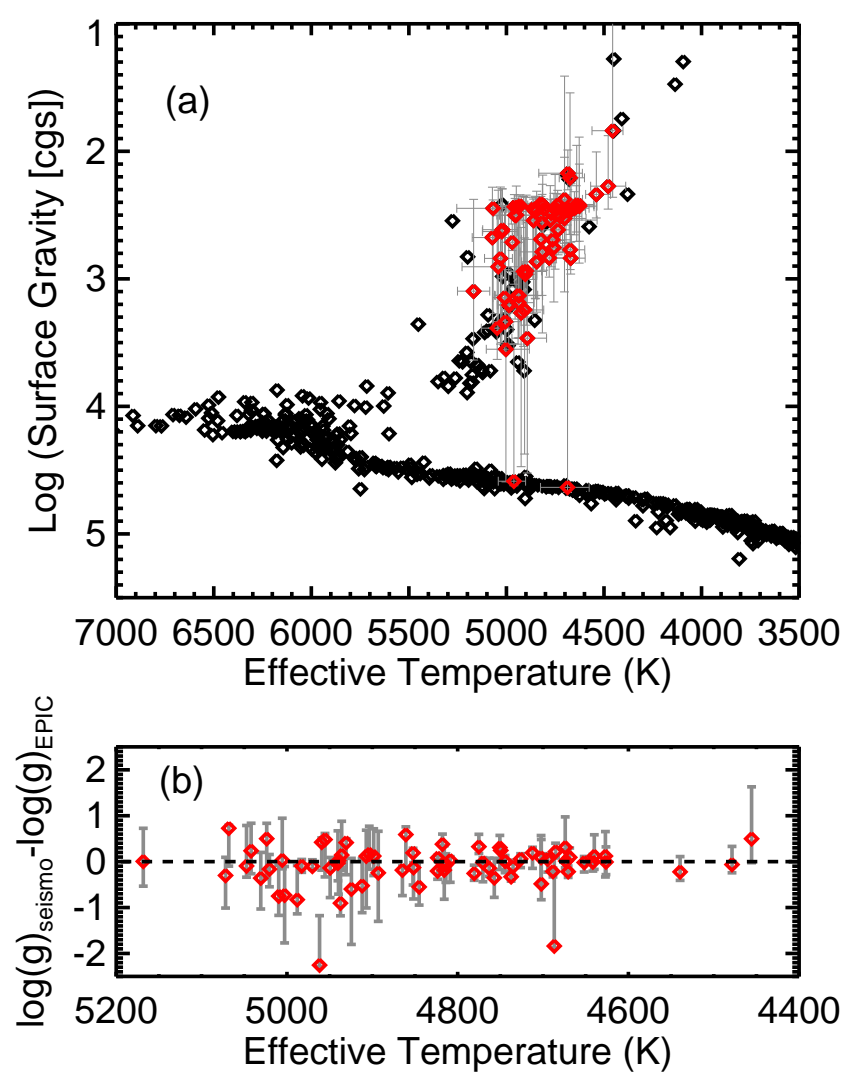

FIG. 12.- (a) Surface gravity versus effective temperature taken from the EPIC for $612 \mathrm{~K} 2$ targets observed in C5. Red triangles with error bars show asteroseismically confirmed giants based on the analysis of K2 data (see Section [5.6). (b) Difference between $\log g$ in the EPIC and $\log g$ derived from asteroseismology. The residual scatter is $\approx 0.35$ dex.

are heavily contaminated by the 6 hour pointing artefacts. The remaining 5 stars are likely dwarfs that are misclassified as giants due to erroneous proper motions.

To test the accuracy of the derived stellar properties we combined the measured frequency of maximum power $\left(\nu_{\max }\right)$ for each oscillating giant with $T_{\text {eff }}$ from the EPIC to calculate $\log g$, and compared these values to those in 
the EPIC in Figure 12b. The residuals shows a scatter of $\approx 0.35$ dex with no systematic offset, confirming that the EPIC classifications do not only discern dwarfs from giants with a low false positive rate, but also have some sensitivity to evolutionary states on the red giant branch.

\subsection{K2 Stellar Properties Catalog and Shortcomings}

Table 5 lists stellar properties, distances and extinction values for the $138,600 \mathrm{~K} 2$ targets in $\mathrm{C} 1-\mathrm{C} 8$ (88\% of the total target sample) derived in this work. Reported values are the posterior median and 1- $\sigma$ confidence interval around the median. Note that for a small fraction $(\approx 0.2 \%)$ of stars with bimodal distributions where the median would result in unphysical stellar properties we adopted the best-fit and 1- $\sigma$ confidence interval around the best fit instead.

The table also includes a flag denoting which observables were used to derive the listed properties. From all 138,600 classified C1-8 targets, 1\% were classified using parallaxes and colors, $7 \%$ using spectroscopy, $81 \%$ using reduced proper motions and colors, and $11 \%$ using colors only. The source code used to derive the classifications is available ${ }^{14}$ and can be used to extract posterior distributions for each target.

We stress that while the catalog should be useful for a first-look classification of K2 targets, care should be taken when using the catalog for scientific investigations. Specifically, the following shortcomings should be expected:

- A significant fraction ( $\approx 55-70 \%)$ of subgiants are misclassified as dwarfs, and hence their radii will be systematically underestimated. This uncertainty will in most cases be captured in the posterior distributions (and hence the quoted confidence intervals), but using point estimates for a large population may lead to biased results.

- By construction, stars which are rare in the Galaxia populations (such as pre-main sequence stars, massive main-sequence stars, AGB stars, and white dwarfs) will either be missing or misclassified in the catalog. Users are encouraged to use the observational information in the EPIC (such as colors) to identify and characterize such sources.

- Metallicities for stars without spectroscopic input are statistical estimates only and should not be used for scientific investigations.

- The reddening model in Galaxia does not take into account low extinction regions in the solar neighborhood (the "local bubble"), and hence $E(B-V)$ values are likely overestimated for stars within $\lesssim 100$ pc (in particular for campaigns close to the galactic plane).

- Uncertainties provided in the catalog do not account for systematic errors in isochrone models such as different physical prescriptions of mixing length, convective core-overshooting, helium abundances, and mass loss. We have adopted the following lower limits for uncertainties: $1 \%$ in $T_{\text {eff }}$,

14 https://github.com/danxhuber/galclassify
0.02 dex in $\log g, 0.05$ dex in $[\mathrm{Fe} / \mathrm{H}], 2 \%$ in radius and mass, $3 \%$ in distance, and $6 \%$ in density.

- Galaxia uses Padova isochrones to predict masses and radii, which show systematic differences to other model grids. In particular, Padova models are known to systematically underpredict radii for cool dwarfs by up to $20 \%$ (Bovajian et al. 2012a). Hence, users are encouraged to apply empirical corrections to radii of cool dwarfs, or use the derived $T_{\text {eff }}$ values with empirical calibrations to estimate radii (Mann et al. 2015). Future revisions of Galaxia will implement improved isochrones to alleviate this problem.

\section{SUMMARY \& CONCLUSIONS}

We presented the Ecliptic Plane Input Catalog (EPIC) for the $\mathrm{K} 2$ mission, which serves a similar purpose as the Kepler Input Catalog (KIC) for the Kepler Mission. The EPIC is a federation of the Hipparcos, Tycho-2, 2MASS, UCAC4 and SDSS catalogs and contains EPIC identifiers, coordinates, Kepler magnitudes, photometry, astrometry and cross-matched identifiers for each source. Kepler magnitudes in the EPIC are accurate to $\approx 0.1 \mathrm{mag}$, except if they are based on $J$-band photometry only for which discrepancies of $0.5 \mathrm{mag}$ or more can be expected. The EPIC is magnitude complete down to $K p \approx 17$ for typical campaigns, and down to $K p \approx 19$ covered by SDSS. For non-SDSS fields the completeness is set by $2 \mathrm{MASS}$, and hence the catalog is expected to be significantly more incomplete for blue objects. We furthermore described the procedures to mitigate missing sources which are proposed for K2 observations, and discussed several known shortcomings (such as duplicate sources and incompleteness) which should be kept in mind when using the EPIC. At the time of writing, the EPIC includes 48 million sources for $\mathrm{C} 0-\mathrm{C} 13$.

Unlike the KIC, the EPIC does not include stellar classifications for each catalogued source. To support the K2 community we have derived stellar properties (temperatures, surface gravities, metallicities, radii, masses, densities, distances, and extinctions) for 138,600 K2 targets in $\mathrm{C} 1-8$ using colors, proper motions, spectroscopy, parallaxes and stellar population models for each field. We demonstrated that these classifications allow a reliable $(\approx 95 \%$ success rate) separation between giants and dwarfs, but suffer from biases to classify $\approx 55-70 \%$ of subgiants as dwarfs. However, this bias should in most cases be appropriately captured in the provided uncertainties. Our tests imply a precision of $\approx 2-3 \%$ for $T_{\text {eff }}$, $\approx 0.3 \mathrm{dex}$ in $\log g$ and $\approx 0.3 \mathrm{dex}$ in $[\mathrm{Fe} / \mathrm{H}]$, which is consistent with typical formal uncertainties for G-type stars in the catalog: $140 \mathrm{~K}$ in $T_{\text {eff }}, \approx 0.3 \mathrm{dex}$ in $\log g, \approx 0.3 \mathrm{dex}$ in $[\mathrm{Fe} / \mathrm{H}], \approx 40 \%$ in radius, $\approx 10 \%$ in mass, $\approx 60 \%$ in density, and $\approx 40 \%$ in distance. We emphasize that the uncertainties vary considerably with spectral type and evolutionary state, and do not take into account systematic errors between different isochrones.

We showed that the K2 target sample in $\mathrm{C} 1-8$ is dominated by $\mathrm{K}-\mathrm{M}$ dwarfs ( $41 \%$ of all classified targets) followed by $\mathrm{F}-\mathrm{G}$ dwarfs (36\%) and $\mathrm{K}$ giants (21\%), consistent with key K2 science programs to detect transiting exoplanets and conduct galactic archeology using oscillating red giants. We showed that these distributions 
TABLE 5

Stellar Properties of K2 Targets

\begin{tabular}{|c|c|c|c|c|c|c|c|c|c|}
\hline EPIC & $\begin{array}{l}T_{\text {eff }} \\
(\mathrm{K})\end{array}$ & $\begin{array}{l}\log g \\
(\mathrm{cgs})\end{array}$ & {$[\mathrm{Fe} / \mathrm{H}]$} & $\begin{array}{c}R \\
\left(R_{\odot}\right) \\
\end{array}$ & $\begin{array}{c}M \\
\left(M_{\odot}\right)\end{array}$ & $\begin{array}{c}\rho \\
\left(\rho_{\odot}\right)\end{array}$ & $\begin{array}{c}\mathrm{d} \\
(\mathrm{pc})\end{array}$ & $\begin{array}{c}\mathrm{E}(\mathrm{B}-\mathrm{V}) \\
(\mathrm{mag})\end{array}$ & Flag \\
\hline 201425819 & $3937_{-70}^{+117}$ & $4.849_{-0.035}^{+0.042}$ & $-0.024_{-0.210}^{+0.120}$ & $0.431_{-0.051}^{+0.035}$ & $0.486_{-0.053}^{+0.053}$ & $5.79_{-0.86}^{+1.47}$ & $202_{-25}^{+23}$ & $0.032_{-0.014}^{+0.014}$ & rpm \\
\hline 201426057 & $3935_{-192}^{+231}$ & $4.864_{-0.075}^{+0.105}$ & $-0.113_{-0.240}^{+0.200}$ & $0.411_{-0.100}^{+0.079}$ & $0.455_{-0.128}^{+0.091}$ & $6.48_{-1.95}^{+4.20}$ & $241_{-68}^{+65}$ & $0.029_{-0.012}^{+0.014}$ & $\mathrm{col}$ \\
\hline 201426077 & $5658_{-135}^{+169}$ & $3.888_{-0.040}^{+0.096}$ & $-0.251_{-0.150}^{+0.150}$ & $1.91_{-0.22}^{+0.150}$ & $1.072_{-0.108}^{+0.068}$ & $0.149_{-0.024}^{+0.054}$ & $1034_{-125}^{+86}$ & $0.028_{-0.008}^{+0.013}$ & lam \\
\hline 201426087 & $4977_{-80}^{+100}$ & $2.774_{-0.600}^{+0.600}$ & $-0.735_{-0.420}^{+0.300}$ & $5.71_{-2.63}^{+6.01}$ & $0.915_{-0.041}^{+0.116}$ & $0.0038_{-0.0033}^{+0.0165}$ & $1736_{-697}^{+1709}$ & $0.029_{-0.010}^{+0.016}$ & rpm \\
\hline 201426089 & $4229_{-153}^{+153}$ & $4.751_{-0.055}^{+0.055}$ & $-0.103_{-0.240}^{+0.160}$ & $0.523_{-0.058}^{+0.065}$ & $0.581_{-0.066}^{+0.071}$ & $3.82_{-0.82}^{+1.04}$ & $510_{-77}^{+92}$ & $0.033_{-0.013}^{+0.016}$ & rpm \\
\hline 201426095 & $6346_{-192}^{+154}$ & $4.226_{-0.170}^{+0.085}$ & $-0.146_{-0.270}^{+0.150}$ & $1.35_{-0.1}^{+0.3}$ & $1.17_{-0.15}^{+0.13}$ & $0.45_{-0.22}^{+0.16}$ & $504_{-66}^{+148}$ & $0.028_{-0.009}^{+0.016}$ & rpm \\
\hline 201426122 & $3712_{-219}^{+263}$ & $4.968_{-0.100}^{+0.100}$ & $-0.103_{-0.280}^{+0.200}$ & $0.290_{-0.085}^{+0.097}$ & $0.31_{-0.12}^{+0.14}$ & $11.03_{-4.53}^{+9.79}$ & $231_{-83}^{+104}$ & $0.027_{-0.010}^{+0.016}$ & col \\
\hline 201426233 & $5859_{-183}^{+114}$ & $4.330_{-0.260}^{+0.130}$ & $-0.133_{-0.240}^{+0.200}$ & $1.11_{-0.20}^{+0.450}$ & $0.99_{-0.10}^{+0.13}$ & $0.67_{-0.43}^{+0.50}$ & $643_{-133}^{+268}$ & $0.033_{-0.014}^{+0.014}$ & $\mathrm{rpm}$ \\
\hline 201426240 & $5657_{-111}^{+111}$ & $4.467_{-0.384}^{+0.072}$ & $-0.324_{-0.350}^{+0.250}$ & $0.90_{-0.11}^{+0.39}$ & $0.888_{-0.089}^{+0.089}$ & $\begin{array}{r}1.19_{-0.73}^{+0.36} \\
1.36 \\
\end{array}$ & $244_{-35}^{+134}$ & $0.029_{-0}^{+0}$ & $\mathrm{rpm}$ \\
\hline 201426279 & $4831_{-176}^{+117}$ & $4.664_{-0.030}^{+0.054}$ & $-0.503_{-0.400}^{+0.250}$ & $0.620_{-0.063}^{+0.049}$ & $0.659_{-0.059}^{+0.059}$ & $2.66_{-0.41}^{+0.75}$ & $443_{-48}^{+46}$ & $0.024_{-0.005}^{+0.025}$ & $\mathrm{rpm}$ \\
\hline 201426280 & $4082_{-48}^{+48}$ & $4.799_{-0.035}^{+0.042}$ & $-0.045_{-0.150}^{+0.150}$ & $0.483_{-0.046}^{+0.036}$ & $0.552_{-0.055}^{+0.039}$ & $4.64_{-0.64}^{+0.91}$ & $517_{-55}^{+-48}$ & $0.036_{-0.015}^{+0.015}$ & $\mathrm{rpm}$ \\
\hline 201426327 & $3786_{-59}^{+59}$ & $4.954_{-0.045}^{+0.063}$ & $-0.191_{-0.100}^{+0.100}$ & $0.334_{-0.069}^{+0.035}$ & $0.365_{-0.094}^{+0.043}$ & $9.70_{-1.92}^{+4.32}$ & $259_{-57}^{+34}$ & $0.044_{-0.021}^{+0.013}$ & rpm \\
\hline 201426389 & $4199_{-99}^{+124}$ & 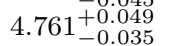 & $-0.073_{-0.240}^{+0.160}$ & $0.523_{-0.054}^{+0.043}$ & $0.573_{-0.048}^{+0.048}$ & $4.01_{-0.60}^{+1.01}$ & $398_{-49}^{+39}$ & $0.030_{-0.011}^{+0.015}$ & rpm \\
\hline 201426415 & $3967_{-191}^{+267}$ & $4.849_{-0.090}^{+0.075}$ & $-0.055_{-0.240}^{+0.150}$ & $0.428_{-0.075}^{+0.091}$ & $0.471_{-0.092}^{+0.111}$ & $5.92_{-2.11}^{+2.64}$ & $272_{-55}^{+85}$ & $0.032_{-0.012}^{+0.011}$ & rpm \\
\hline 201426494 & $4115_{-237}^{+237}$ & $4.803_{-0.080}^{+0.080}$ & $-0.143_{-0.240}^{+0.200}$ & $0.469_{-0.085}^{+0.103}$ & $0.536_{-0.088}^{+0.088}$ & $4.71_{-1.38}^{+1.94}$ & $453_{-98}^{+125}$ & $0.032_{-0.012}^{+0.017}$ & rpm \\
\hline 201426531 & $3868_{-38}^{+38}$ & $4.898_{-0.030}^{+0.020}$ & $-0.140_{-0.050}^{+0.100}$ & $0.381_{-0.011}^{+0.033}$ & $0.423_{-0.014}^{+0.041}$ & $7.61_{-1.13}^{+0.46}$ & $270_{-12}^{+24}$ & $0.057_{-0.018}^{+0.001}$ & rpm \\
\hline 201426541 & $3551_{-42}^{+35}$ & $5.021_{-0.042}^{+0.036}$ & $0.044_{-0.160}^{+0.200}$ & $0.254_{-0.020}^{+0.036}$ & $0.248_{-0.018}^{+0.046}$ & $15.26_{-3.36}^{+2.26}$ & $144_{-11}^{+20}$ & $0.023_{-0.005}^{+0.015}$ & rpm \\
\hline 201426580 & $5179_{-82}^{+144}$ & $3.236_{-0.990}^{+0.693}$ & $-0.949_{-1.440}^{+0.400}$ & $\begin{array}{l}3.21_{-2.09}^{+6.98} \\
x^{2}\end{array}$ & $0.876_{-0.077}^{+0.026}$ & $0.018_{-0.018}^{+0.172}$ & $2156_{-988}^{+4483}$ & $0.030_{-0.012}^{+0.014}$ & rpm \\
\hline 201426597 & $3872_{-46}^{+46}$ & $4.893_{-0.035}^{+0.049}$ & $-0.113_{-0.120}^{+0.120}$ & $0.383_{-0.047}^{+0.038}$ & $0.433_{-0.056}^{+0.046}$ & $7.33_{-1.16}^{+2.010}$ & $333_{-47}^{+38}$ & $0.035_{-0.013}^{+0.013}$ & rpm \\
\hline 201426615 & $3748_{-322}^{+386}$ & $4.959_{-0.156}^{+0.156}$ & $-0.133_{-0.280}^{+0.200}$ & $0.283_{-0.097}^{+0.147}$ & $0.32_{-0.15}^{+0.18}$ & $10.82_{-5.76}^{+12.31}$ & $409_{-193}^{+288}$ & $0.029_{-0.012}^{+0.017}$ & $\mathrm{col}$ \\
\hline & & & & & & 前 & & & \\
\hline
\end{tabular}

Notes: Reported values are the posterior median and 1- $\sigma$ confidence interval around the median. Flags indicate which observables were used to derive the classification: hip = Hipparcos parallax + colors; apo = APOGEE spectroscopic classification; rav $=$ RAVE spectroscopic classification; lam = LAMOST spectroscopic classification; $\mathrm{rpm}=$ reduced proper motions + colors; col = colors only;

vary considerably over different campaigns: low galactic latitude fields appear to have a larger relative fraction of giants, presumably as a result of the increased giant contamination and reddening introducing a bias in the target selection. We used a set of asteroseismic giants to verify the giant-dwarf classifications in the catalog but also find significant differences to spectroscopic classifications of several K2 exoplanet host stars, which emphasize the need for detailed spectroscopic follow-up to reliably characterize detected transiting planets. Future observations from large-scale spectroscopic surveys such as APOGEE (Allende Prieto et al. 2008) and GALAH (De Silva et al. 2015) as well as parallaxes from Gaia (Perryman 2005) will considerably improve the classifications provided here.

The EPIC is hosted at the Mikulski Archive for Space Telescopes (MAST) 15 and EPIC information on K2 targets (including stellar classifications) and confirmed planet hosts can be found at the NASA Exoplanet Archive 16 . The source codes to produce an EPIC 17 and to derive stellar classifications (including posteriors) for K2 target:18 are publicly available. We note that the EPIC is continuously updated for new K2 campaign fields, and users are encouraged to regularly check the online documentation at MAST 19 for updates.

\section{ACKNOWLEDGMENTS}

\footnotetext{
15 http://archive.stsci.edu/k2/epic/search.php

16 http://exoplanetarchive.ipac.caltech.edu/

17 https://github.com/danxhuber/k2epic

18 https://github.com/danxhuber/galclassify

19 http://archive.stsci.edu/k2/epic.pdf
}

We thank Tim Bedding, Jeff van Cleve and Sebastien Lepine for comments and discussions, and numerous members of the K2 community for valuable feedback and bug reports. We also thank Randy Thompson, Scott Fleming and everyone at MAST for their help in making the EPIC available to the community. We are furthermore grateful for the support of the Kavli Institute for Theoretical Physics in Santa Barbara, where part of this research was conducted.

D.H. acknowledges support by the Australian Research Council's Discovery Projects funding scheme (project number DE140101364) and support by the National Aeronautics and Space Administration under Grant NNX14AB92G issued through the Kepler Participating Scientist Program. Funding for the Kepler Mission is provided by NASA's Science Mission Directorate. This publication makes use of data products from the Two Micron All Sky Survey, which is a joint project of the University of Massachusetts and the Infrared Processing and Analysis Center/California Institute of Technology, funded by the National Aeronautics and Space Administration and the National Science Foundation. This research made use of the AAVSO Photometric All-Sky Survey (APASS), funded by the Robert Martin Ayers Sciences Fund. The Second Palomar Observatory Sky Survey (POSS-II) was made by the California Institute of Technology with funds from the National Science Foundation, the National Geographic Society, the Sloan Foundation, the Samuel Oschin Foundation, and the Eastman Kodak Corporation. This research was supported in part by the National Science Foundation under Grant No. NSF PHY11-25915. 


\section{REFERENCES}

Ahn, C. P., Alexandroff, R., Allende Prieto, C., et al. 2012, ApJS, 203, 21

Aigrain, S., Hodgkin, S. T., Irwin, M. J., Lewis, J. R., \& Roberts, S. J. 2015, MNRAS, 447, 2880

Alam, S., Albareti, F. D., Allende Prieto, C., et al. 2015, ApJS, 219, 12

Allende Prieto, C., Majewski, S. R., Schiavon, R., et al. 2008, Astronomische Nachrichten, 329, 1018

Angus, R., Foreman-Mackey, D., \& Johnson, J. A. 2016, ApJ, 818, 109

Armstrong, D. J., Kirk, J., Lam, K. W. F., et al. 2015, A\&A, 579, A19

Batalha, N. M., Borucki, W. J., Koch, D. G., et al. 2010, ApJ, 713, L109

Batalha, N. M., Rowe, J. F., Bryson, S. T., et al. 2013, ApJS, 204, 24

Bessell, M. S. 2000, PASP, 112, 961

Bilir, S., Ak, S., Karaali, S., et al. 2008, MNRAS, 384, 1178

Bilir, S., Karaali, S., \& Tunçel, S. 2005, Astronomische Nachrichten, 326, 32

Borucki, W. J., Koch, D., Basri, G., et al. 2010, Science, 327, 977

Borucki, W. J., Koch, D. G., Basri, G., et al. 2011a, ApJ, 728, 117

-. 2011b, ApJ, 736, 19

Boyajian, T. S., McAlister, H. A., van Belle, G., et al. 2012a, ApJ, 746, 101

Boyajian, T. S., von Braun, K., van Belle, G., et al. 2012b, ApJ, 757,112

-. 2013, ApJ, 771, 40

Bressan, A., Marigo, P., Girardi, L., et al. 2012, MNRAS, 427, 127

Brown, T. M., Latham, D. W., Everett, M. E., \& Esquerdo, G. A. 2011, AJ, 142, 112

Bruntt, H., Bedding, T. R., Quirion, P.-O., et al. 2010, MNRAS, 405, 1907

Buchhave, L. A., Latham, D. W., Johansen, A., et al. 2012, Nature, 486, 375

Buchhave, L. A., Bizzarro, M., Latham, D. W., et al. 2014, Nature, 509, 593

Buysschaert, B., Aerts, C., Bloemen, S., et al. 2015, MNRAS, 453, 89

Carter, J. A., Fabrycky, D. C., Ragozzine, D., et al. 2011 Science, 331, 562

Casagrande, L., Schönrich, R., Asplund, M., et al. 2011, A\&A, 530, A138

Casagrande, L., Silva Aguirre, V., Stello, D., et al. 2014, ApJ, 787,110

Chaplin, W. J., Kjeldsen, H., Christensen-Dalsgaard, J., et al. 2011, Science, 332, 213

Chaplin, W. J., Lund, M. N., Handberg, R., et al. 2015, PASP, 127,1038

Crossfield, I. J. M., Petigura, E., Schlieder, J. E., et al. 2015, ApJ, 804, 10

De Silva, G. M., Freeman, K. C., Bland-Hawthorn, J., et al. 2015 MNRAS, 449, 2604

Dotter, A., Chaboyer, B., Jevremović, D., et al. 2008, ApJS, 178, 89

Foreman-Mackey, D., Montet, B. T., Hogg, D. W., et al. 2015, ApJ, 806, 215

Fressin, F., Torres, G., Charbonneau, D., et al. 2013, ApJ, 766, 81

Girardi, L., Bressan, A., Bertelli, G., \& Chiosi, C. 2000, A\&AS, 141,371

Gould, A., \& Morgan, C. W. 2003, ApJ, 585, 1056

Henderson, C. B., Penny, M., Street, R. A., et al. 2015, ArXiv e-prints, arXiv: 1512.09142

Hermes, J. J., Charpinet, S., Barclay, T., et al. 2014, ApJ, 789, 85

Høg, E., Fabricius, C., Makarov, V. V., et al. 2000, A\&A, 355, L27

Howard, A. W., Marcy, G. W., Bryson, S. T., et al. 2012, ApJS, 201,15

Howell, S. B., Rowe, J. F., Bryson, S. T., et al. 2012, ApJ, 746,

Howell, S. B., Sobeck, C., Haas, M., et al. 2014, PASP, 126, 398

Huang, C. X., Penev, K., Hartman, J. D., et al. 2015, MNRAS, 454,4159

Huber, D., Stello, D., Bedding, T. R., et al. 2009,

Communications in Asteroseismology, 160, 74
Huber, D., Chaplin, W. J., Christensen-Dalsgaard, J., et al. 2013 ApJ, 767, 127

Huber, D., Silva Aguirre, V., Matthews, J. M., et al. 2014, ApJS, 211,2

Jeffery, C. S., \& Ramsay, G. 2014, MNRAS, 442, L61

Johnson, D. R. H., \& Soderblom, D. R. 1987, AJ, 93, 864

Kallinger, T., Mosser, B., Hekker, S., et al. 2010, A\&A, 522, A1

Koch, D. G., Borucki, W. J., Basri, G., et al. 2010, ApJ, 713, L79

Kordopatis, G., Gilmore, G., Steinmetz, M., et al. 2013, AJ, 146, 134

Kraus, A. L., Tucker, R. A., Thompson, M. I., Craine, E. R., \& Hillenbrand, L. A. 2011, ApJ, 728, 48

Kurtz, D. W., Bowman, D. M., Ebo, S. J., et al. 2016, MNRAS, 455,1237

LaCourse, D. M., Jek, K. J., Jacobs, T. L., et al. 2015, MNRAS, 452,3561

Lund, M. N., Handberg, R., Davies, G. R., Chaplin, W. J., \& Jones, C. D. 2015, ApJ, 806, 30

Luo, A.-L., Zhao, Y.-H., Zhao, G., et al. 2015, Research in Astronomy and Astrophysics, 15, 1095

Mann, A. W., Feiden, G. A., Gaidos, E., Boyajian, T., \& von Braun, K. 2015, ApJ, 804, 64

Marigo, P., \& Girardi, L. 2007, A\&A, 469, 239

Marigo, P., Girardi, L., Bressan, A., et al. 2008, A\&A, 482, 883

Miglio, A., Chiappini, C., Morel, T., et al. 2013, MNRAS, 429, 423

Molnár, L., Szabó, R., Moskalik, P. A., et al. 2015, MNRAS, 452, 4283

Montet, B. T., Morton, T. D., Foreman-Mackey, D., et al. 2015, ApJ, 809, 25

Morton, T. D. 2015, isochrones: Stellar model grid package, Astrophysics Source Code Library, , , ascl:1503.010

Perryman, M. A. C. 2005, in Astronomical Society of the Pacific Conference Series, Vol. 338, Astrometry in the Age of the Next Generation of Large Telescopes, ed. P. K. Seidelmann \& A. K. B. Monet, 3

Petigura, E. 2015, PhD Thesis, University of California, arXiv:1510.03902

Petigura, E. A., Schlieder, J. E., Crossfield, I. J. M., et al. 2015, ApJ, 811, 102

Pinsonneault, M. H., An, D., Molenda-Żakowicz, J., et al. 2012, ApJS, 199, 30

Pinsonneault, M. H., Elsworth, Y., Epstein, C., et al. 2014, ApJS, 215, 19

Robin, A. C., Reylé, C., Derrière, S., \& Picaud, S. 2003, A\&A, 409, 523

Sanchis-Ojeda, R., Rappaport, S., Pallè, E., et al. 2015, ApJ, 812, 112

Schlegel, D. J., Finkbeiner, D. P., \& Davis, M. 1998, ApJ, 500, 525

Schlieder, J. E., Crossfield, I. J. M., Petigura, E. A., et al. 2016, ApJ, 818, 87

Serenelli, A. M., Bergemann, M., Ruchti, G., \& Casagrande, L. 2013, MNRAS, 429, 3645

Sharma, S., Bland-Hawthorn, J., Johnston, K. V., \& Binney, J. 2011, ApJ, 730, 3

Sharma, S., Bland-Hawthorn, J., Binney, J., et al. 2014, ApJ, 793,51

Sinukoff, E., Howard, A. W., Petigura, E. A., et al. 2015, ApJ, submitted, arXiv:1511.09213

Skrutskie, M. F., Cutri, R. M., Stiening, R., et al. 2006, AJ, 131, 1163

Stassun, K. G., Pepper, J. A., Paegert, M., De Lee, N., \& Sanchis-Ojeda, R. 2014, ArXiv e-prints, arXiv:1410.6379

Stello, D., Huber, D., Sharma, S., et al. 2015, ApJ, 809, L3

Thompson, S. E., \& Fraquelli, D. 2012, Kepler Archive Manual (KDMC-10008-003), http://archive.stsci.edu/kepler/documents.html

van Leeuwen, F. 2007, A\&A, 474, 653

Vanderburg, A., \& Johnson, J. A. 2014, PASP, 126, 948

Vanderburg, A., Montet, B. T., Johnson, J. A., et al. 2015, ApJ, 800,59

Vanderburg, A., Latham, D. W., Buchhave, L. A., et al. 2016, ApJS, 222, 14 
Verner, G. A., Elsworth, Y., Chaplin, W. J., et al. 2011, MNRAS, 415,3539

Youdin, A. N. 2011, ApJ, 742, 38
Zacharias, N., Finch, C. T., Girard, T. M., et al. 2013, AJ, 145, 44 Zacharias, N., Monet, D. G., Levine, S. E., et al. 2005, VizieR Online Data Catalog, 1297, 0 